\title{
ITERATED SPECTRA OF NUMBERS-ELEMENTARY, DYNAMICAL, AND ALGEBRAIC APPROACHES
}

\author{
VITALY BERGELSON, NEIL HINDMAN, AND BRYNA KRA
}

\begin{abstract}
I $P^{*}$ sets and central sets are subsets of $\mathbb{N}$ which arise out of applications of topological dynamics to number theory and are known to have rich combinatorial structure. Spectra of numbers are often studied sets of the form $\{[n \alpha+\gamma]: n \in \mathbb{N}\}$. Iterated spectra are similarly defined with $n$ coming from another spectrum. Using elementary, dynamical, and algebraic approaches we show that iterated spectra have significantly richer combinatorial structure than was previously known. For example we show that if $\alpha>0$ and $0<\gamma<1$, then $\{[n \alpha+\gamma]: n \in \mathbb{N}\}$ is an $I P^{*}$ set and consequently contains an infinite sequence together with all finite sums and products of terms from that sequence without repetition.
\end{abstract}

\section{INTRODUCTION}

A $\Delta$ set is a subset of the set $\mathbb{N}$ of positive integers which contains an infinite difference set, that is, a set of the form $\{x-y: x, y \in A$ and $y<x\}$ for some infinite $A \subset \mathbb{N}$. An $I P$ set is a set containing $\operatorname{FS}\left(\left\langle x_{n}\right\rangle_{n=1}^{\infty}\right)$ for some infinite sequence $\left\langle x_{n}\right\rangle_{n=1}^{\infty}$ in $\mathbb{N}$ (where the set of finite sums of the sequence $\left\langle x_{n}\right\rangle_{n=1}^{\infty}, \operatorname{FS}\left(\left\langle x_{n}\right\rangle_{n=1}^{\infty}\right)=$ $\left\{\sum_{n \in F} x_{n}: F\right.$ is a finite nonempty subset of $\left.\mathbb{N}\right\}$ ). (The terminology in both cases differs from [12] in that for us both notions are closed under supersets. They are notions of combinatorial richness, so bigger ought to be better.)

Our third basic notion of combinatorial richness, namely the notion of central sets, was introduced in [12] with a definition in terms of topological dynamics. This definition can be found in Section 3. An alternate, algebraic, characterization of "central" was shown in [5] to be equivalent: A subset $A$ of $\mathbb{N}$ is central if and only if there is a minimal idempotent $p$ of $(\beta \mathbb{N},+)$ with $A \in p$. (Here $\beta \mathbb{N}$ is the StoneCech compactification of $\mathbb{N}$ and + denotes the extension of ordinary addition to $\beta \mathbb{N}$ which makes $(\beta \mathbb{N},+)$ a left topological semigroup with $\mathbb{N}$ contained in its center.) An element $p$ of $\beta \mathbb{N}$ is a minimal idempotent provided $p=p+p$ and $p$ is a member of some minimal right ideal of $\beta \mathbb{N}$. (A right ideal $R$ satisfies $R+\beta \mathbb{N} \subset \beta \mathbb{N}$.) We will describe this structure in more detail later in this introduction.

Each central set is an $I P$ set and in fact has very intricate combinatorial structure including arbitrarily long arithmetic progressions ([12, Proposition 8.21] or see [5]). To see that each $I P$ set is a $\Delta$ set let $\operatorname{FS}\left(\left\langle x_{n}\right\rangle_{n=1}^{\infty}\right) \subseteq A$ and consider the differences from $\left\{\sum_{k=1}^{n} x_{k}: n \in \mathbb{N}\right\}$.

Received by the editors November 5, 1994.

1991 Mathematics Subject Classification. Primary 05D10, Secondary 22A15, 54H20, 05B10.

The first two author gratefully acknowledge support received from the National Science Foundation (USA) via grants DMS-9401093 and DMS-9424421 respectively. 
Given any class $\mathcal{B}$ of subsets of $\mathbb{N}$, there is a corresponding class $\mathcal{B}^{*}$ defined by: $A \in \mathcal{B}^{*}$ if and only if for each $B \in \mathcal{B}, A \cap B \neq \emptyset$. Obviously then if $\mathcal{B} \subseteq \mathcal{C}$ one has $\mathcal{C}^{*} \subseteq \mathcal{B}^{*}$. Further, if $\mathbb{N} \in \mathcal{B}$ and $\mathcal{B}$ is partition regular (meaning that whenever the union of finitely many sets is in $\mathcal{B}$, some one of them is), then $\mathcal{B}^{*} \subseteq \mathcal{B}$. (To see this let $A \in \mathcal{B}^{*}$. Then either $A$ or $\mathbb{N} \backslash A$ is in $\mathcal{B}$ and $A \cap(\mathbb{N} \backslash A)=\emptyset$ so $A \in \mathcal{B}$.) Since the notion of "central" is partition regular, we have $\Delta^{*} \subseteq I P^{*} \subseteq$ central $^{*} \subseteq$ central $\subseteq I P \subseteq \Delta$. (According to [12, p. 178 and p. 186] all these inclusions are proper.)

Since central sets are partition regular, explicit examples of central sets are plentiful. That is, define any finite partition of $\mathbb{N}$. At least one cell is guaranteed to be central. For example, partition $\mathbb{N}$ according to the rightmost nonzero ternary digit of each $x \in \mathbb{N}$. (So $A_{1}=\left\{3^{k}(3 m+1): k, m \in \mathbb{N} \cup\{0\}\right\}, A_{2}=$ $\left\{3^{k}(3 m+2): k, m \in \mathbb{N} \cup\{0\}\right\}$. Then either $A_{1}$ or $A_{2}$ must be central. But it is easy to see that if $B$ is central so is $2 \cdot B$. (See for instance Lemma 3.8(b).) So we must have that both $A_{1}$ and $A_{2}$ are central. Many more explicit examples are produced in this paper.

Further, it is possible to partition $\mathbb{N}$ into infinitely many parts, each of which is central [5, Corollary 5.9].

Consider, on the other hand, $\Delta^{*}$ sets and $I P^{*}$ sets. (The structure of $I P^{*}$ sets is known to be very rich - see [6].) It is easy to see that for any $n \in \mathbb{N}$, the set $\mathbb{N} n$ of multiples of $n$ is a $\Delta^{*}$-set, and hence an $I P^{*}$ set. (Given any infinite set some 2 elements are congruent $\bmod n$ and their difference is divisible by $n$.) It is also known for example [12, Proposition 9.4] that if $(X, T)$ is a minimal compact metric dynamical system (that is, no proper nonempty closed subset of $X$ is invariant under $T), l \in \mathbb{N}$, and $U$ is a nonempty open subset of $X$, then $\left\{n \in \mathbb{N}: U \cap T^{-n} U \cap\right.$ $\left.\cdots \cap T^{-l n} U \neq \emptyset\right\}$ is an $I P^{*}$ set. Other explicit examples of $I P^{*}$ sets are deducible from [13]. However, there would seem to be a shortage of simply described $\Delta^{*}$ sets and $I P^{*}$ sets. It is in this context that we are interested in the spectra of numbers.

Given an irrational number $\alpha>1$ and $\delta=\alpha /(\alpha-1)$ (so that $1 / \alpha+1 / \delta=1$ ) it is "an often rediscovered result" $[27$, p. 45] that $\{[n \alpha]: n \in \mathbb{N}\}$ and $\{[n \delta]: n \in \mathbb{N}\}$ are complementary sets. This result may be due originally to J. W. Strutt (Lord Rayleigh) [31]. See [28] for an interesting discussion of the context of this discovery and additional references. Further, Uspensky showed [32] that one cannot partition $\mathbb{N}$ into 3 parts with such sets. (The proof was simplified by Graham in [15], and again by Fraenkel in [11]. In fact there is an even simpler proof that if $\alpha_{1}, \alpha_{2}, \alpha_{3}$ are positive, then $\left\{\left[n \alpha_{1}\right]: n \in \mathbb{N}\right\},\left\{\left[n \alpha_{2}\right]: n \in \mathbb{N}\right\}$, and $\left\{\left[n \alpha_{3}\right]: n \in \mathbb{N}\right\}$ cannot be pairwise disjoint. To see this one utilizes the well known fact [17, Theorem 201] that any set of numbers may be simultaneously brought close to zero mod 1 . Let $\varepsilon=1 / 2\left(\alpha_{1}+\alpha_{2}+\alpha_{3}\right)$ and pick $n, m_{1}, m_{2}$, and $m_{3}$ in $\mathbb{N}$ with $\left|n \cdot 1 / \alpha_{i}-m_{i}\right|<\varepsilon$ for each $i$. Then $\left|n-m_{i} \cdot \alpha_{i}\right|<\varepsilon \cdot \alpha_{i}<1 / 2$ for each $i$, so for each $i,\left[m_{i} \alpha_{i}\right]=n$ or $\left[m_{i} \alpha_{i}\right]=n=1$.)

Numerous results about the sets $\{[n \alpha]: n \in \mathbb{N}\}$ were derived by Skolem [29] and Bang [3] and are nicely presented in [27]. In [30] Skolem introduced the more general sets $\{[n \alpha+\gamma]: n \in \mathbb{N}\}$, determining for example when two such sets can be disjoint.

In terminology introduced by Graham, Lin, and Lin [16], the set $\{[n \alpha+\gamma]: n \in$ $\mathbb{N}\}$ is called the $\gamma$-nonhomogeneous spectrum of $\alpha$. (See also [8].) We shall restrict our attention to such spectra for $0 \leq \gamma \leq 1$. We determine for example that if $0<\gamma<1$, then $\{[n \alpha+\gamma]: n \in \mathbb{N}\}$ is a $\Delta^{*}$ set and consequently enjoys the combinatorial structure guaranteed to such sets. In fact we show much more. For 
example, we have as a consequence of Corollary 2.6 that if $A$ is any $\Delta^{*}$ set (for instance another spectrum), then $\{[n \alpha+\gamma]: n \in A\}$ is again a $\Delta^{*}$ set. In particular if $\alpha_{1}, \alpha_{2}$ are positive and $0<\gamma_{1}<1$ and $0<\gamma_{2}<1$, then $\left\{\left[\left[n \alpha_{1}+\gamma_{1}\right] \alpha_{2}+\gamma_{2}\right]: n \in \mathbb{N}\right\}$ is a $\Delta^{*}$ set. We also show for example as a consequence of Theorem 6.3 that if $\alpha$ is irrational, then both $\{[n \alpha+0]: n \in \mathbb{N}\}$ and $\{[n \alpha+1]: n \in \mathbb{N}\}$ are central. If $\alpha>2$, these sets are disjoint and since each contains $\operatorname{FS}\left(\left\langle x_{n}\right\rangle_{n=1}^{\infty}\right)$ for some sequence, neither is $I P^{*}$. We determine precisely in Theorem 6.7 when the composition of two such sets yields a central set.

Most of the results which we present were first obtained using algebraic methods in $\beta \mathbb{N}$. Subsequently [25] elementary and dynamical proofs of several of these results were obtained, as well as proofs of some additional results. We present our results by the methods we feel best suited for their proofs. In Section 2 we present the results for which we have elementary proofs. (The proofs are elementary both in the intuitive sense and in the fact that they avoid the axiom of choice.)

In Section 3 we present the required background material in Topological Dynamics and in Section 4 present some results on spectra for which we have dynamical proofs. In Section 5 we present the required algebraic background material and present in Section 6 algebraic proofs of several results, concentrating on results about iterated spectra.

We use the semigroup $(\beta \mathbb{N},+)$ where $\beta \mathbb{N}$ is the Stone-Čech compactification of $\mathbb{N}$ and + denotes the extension of ordinary addition to $\beta \mathbb{N}$ which makes $(\beta \mathbb{N},+)$ a left topological semigroup with $\mathbb{N}$ contained in its center. An element $p$ of $\beta \mathbb{N}$ is a minimal idempotent provided $p=p+p$ and $p$ is a member of some minimal right ideal of $\beta \mathbb{N}$. (A right ideal $R$ satisfies $R+\beta \mathbb{N} \subset \beta \mathbb{N}$.)

We take the points of $\beta \mathbb{N}$ to be the ultrafilters on $\mathbb{N}$, the principal ultrafilters being identified with the points of $\mathbb{N}$. Given $A \subseteq \mathbb{N}, \bar{A}=\{p \in \beta \mathbb{N}: A \in p\}$. The set $\{\bar{A}: A \subseteq \mathbb{N}\}$ is a basis for the open sets (as well as a basis for the closed sets) of $\beta \mathbb{N}$. When we say $(\beta \mathbb{N},+)$ is a left topological semigroup, we mean that for each $p \in \beta \mathbb{N}$ the function $\lambda_{p}: \beta \mathbb{N} \rightarrow \beta \mathbb{N}$, defined by $\lambda_{p}(q)=p+q$, is continuous. The operation + on $\beta \mathbb{N}$ is characterized as follows: Given $A \subseteq \mathbb{N}, A \in p+q$ if and only if $\{x \in \mathbb{N}: A-x \in p\} \in q$ where $A-x=\{y \in \mathbb{N}: y+x \in A\}$. See [22] for a detailed construction of $\beta \mathbb{N}$ and derivations of some of the basic algebraic facts.

\section{ElEmentary Results}

We take the circle group $T=\mathbb{R} / \mathbb{Z}$ to be represented either as $[0,1)$ or $\left[-\frac{1}{2}, \frac{1}{2}\right)$, whichever is more convenient.

2.1. Definition. Let $\alpha>0$ and let $h_{\alpha}(n)=\left[n \alpha+\frac{1}{2}\right]$, the nearest integer to $n \alpha$. Define $f_{\alpha}: \mathbb{Z} \rightarrow \mathbf{T}$ by $f_{\alpha}(n)=n \alpha-[n \alpha]$.

In Definition 2.1 we viewed $T$ as $[0,1)$, so that $f_{\alpha}(n)$ is the fractional part of $n \alpha$. If we view $\mathbf{T}$ as $\left[-\frac{1}{2}, \frac{1}{2}\right)$, we have $f_{\alpha}(n)=n \alpha-h_{\alpha}(n)$.

We say that a set $\mathcal{B}$ of subsets of $\mathbb{N}$ is "partition regular" provided that whenever $\mathcal{F}$ is a finite set of subsets of $\mathbb{N}$ with $\bigcup \mathcal{F} \in \mathcal{B}$ one has some $A \in \mathcal{F} \cap \mathcal{B}$.

2.2. Lemma. Let $\mathcal{B}$ be a set of subsets of $\mathbb{N}$ such that:

(1) $\mathcal{B}$ is partition regular.

(2) For each $A \in \mathcal{B}$, there exists $x, y \in A$ with $x+y \in A$.

Then for each $\alpha>0$, each $\varepsilon>0$, and each $A \in \mathcal{B}$, there exists $B \in \mathcal{B}$ such that

$B \subseteq A$ and for all $n \in B, f_{\alpha}(n) \in(-\varepsilon, \varepsilon)$, that is, $-\varepsilon<n \alpha-h_{\alpha}(n)<\varepsilon$. 
Proof. Let $\alpha>0, \varepsilon>0$, and $A \in \mathcal{B}$ be given. Pick $m \in \mathbb{N}$ such that $1 /(2 m)<\varepsilon$ and $m>2$. Let $A_{1}=\left\{n \in A:-1 /(2 m) \leq n \alpha-h_{\alpha}(n)<1 /(2 m)\right\}$ and for $i \in\{2,3, \ldots, 2 m-1\}$ let $A_{i}=\{n \in A:(i-1) /(2 m) \leq n \alpha-[n \alpha]<i / 2 m\}$. Then $A=\bigcup_{i=1}^{2 m-1} A_{i}$ so some $A_{i} \in \mathcal{B}$ by (1). Let $x$ and $y$ be elements of $A_{i}$. If $2 \leq i \leq m-1$, then $x+y \in A_{2 i-1} \cup A_{2 i}$. If $i=m$, then $x+y \in A_{2 m-1} \cup A_{1}$. If $m+1 \leq i \leq 2 m-1$, then $x+y \in A_{2(i-m)-1} \cup A_{2(i-m)}$. In any of these cases $x+y \notin A_{i}$ and hence by condition (2) we must have $i=1$. Since $1 /(2 m)<\varepsilon$, we have, for all $n \in A_{1}, f_{\alpha}(n) \in(-\varepsilon, \varepsilon)$ as required.

We observe that the classes of central sets, IP sets, and $\Delta$ sets all satisfy the hypotheses of Lemma 2.2. Indeed given a $\Delta$ set $A$ and $x, y$, and $z$ with $y-x$, $z-x$, and $z-y$ in $A$ one has $(z-y)+(y-x)=z-x \in A$ so condition (2) holds for $\Delta$ sets and hence for central sets and $I P$ sets. Further each of the classes is known by elementary means to be partition regular. The original proof that $I P$ sets are partition regular [18], while the most complicated of the proofs now in existence, is elementary. The proof that $\Delta$ sets are partition regular is a simple application of Ramsey's Theorem for two element sets, and the standard proofs of Ramsey's Theorem are elementary. The fact that central sets are partition regular is immediate from the algebraic characterization (since if the union of finitely many sets is a member of an ultrafilter, some one of them is a member).

2.3. Theorem. Let $\alpha>0$, let $0<\gamma<1$, and let $A \subseteq \mathbb{N}$.

(a) If $A$ is a $\Delta$ set, then $\{[n \alpha+\gamma]: n \in A\}$ is a $\Delta$ set.

(b) If $A$ is an IP set, then $\{[n \alpha+\gamma]: n \in A\}$ is an IP set.

Proof. Both proofs are similar; we present the proof of (b).

Let $\varepsilon=\min \{\gamma / 2,(1-\gamma) / 2\}$. Pick by Lemma 2.2 some $I P$ set $B \subseteq A$ with $f_{\alpha}(n) \in(-\varepsilon, \varepsilon)$ for all $n \in B$. Observe that for any $n \in B$ one has $h_{\alpha}(n)=[n \alpha+\gamma]$. Indeed $-\gamma<-\varepsilon<n \alpha-h_{\alpha}(n)<\varepsilon<1-\gamma$ so $h_{\alpha}(n)<n \alpha+\gamma<h_{\alpha}(n)+1$. Observe also that for $n, m \in B$ one has $h_{\alpha}(n+m)=h_{\alpha}(n)+h_{\alpha}(m)$ since $\varepsilon \leq 1 / 4$.

Pick a sequence $\left\langle x_{n}\right\rangle_{n=1}^{\infty}$ with $\operatorname{FS}\left(\left\langle x_{n}\right\rangle_{n=1}^{\infty}\right) \subseteq B$. We show by induction on $|F|$ that, for a finite subset $F$ of $\mathbb{N}, \sum_{n \in F} h_{\alpha}\left(x_{n}\right)=h_{\alpha}\left(\sum_{n \in F} x_{n}\right)$. Since $h_{\alpha}\left(\sum_{n \in F} x_{n}\right)$ $=\left[\left(\sum_{n \in F} x_{n}\right) \alpha+\gamma\right]$, this establishes that $\operatorname{FS}\left(\left\langle h_{\alpha}\left(x_{n}\right)\right\rangle_{n=1}^{\infty}\right) \subseteq\{[m \alpha+\gamma]: m \in A\}$.

If $|F|=1$, the conclusion is trivial so assume $|F|>1$, pick $m \in F$, and let $G=F \backslash\{m\}$. Then $\sum_{n \in F} h_{\alpha}\left(x_{n}\right)=h_{\alpha}\left(x_{m}\right)+\sum_{n \in G} h_{\alpha}\left(x_{n}\right)=h_{\alpha}\left(x_{m}\right)+$ $h_{\alpha}\left(\sum_{n \in G} x_{n}\right)=h_{\alpha}\left(x_{m}+\sum_{n \in G} x_{n}\right)$ since $x_{m}$ and $\sum_{n \in G} x_{n}$ are in $B$.

2.4. Theorem. Let $\alpha>0$ with $\alpha \in \mathbb{Q}$ and let $A \subseteq \mathbb{N}$.

(a) If $A$ is a $\Delta$ set, then $\{[n \alpha]: n \in A\}$ is a $\Delta$ set.

(b) If $A$ is an IP set, then $\{[n \alpha]: n \in A\}$ is an IP set.

Proof. Assume $\alpha=p / q$ with $p, q \in \mathbb{N}$. We again present only the proof of (b).

Pick $\left\langle x_{n}\right\rangle_{n=1}^{\infty}$ with $\operatorname{FS}\left(\left\langle x_{n}\right\rangle_{n=1}^{\infty}\right) \subseteq A$. By the pigeon hole principle one may presume $x_{n} \equiv x_{m}(\bmod q)$ for all $n, m \in \mathbb{N}$. Letting $y_{n}=\sum_{t=(n-1) \cdot q+1}^{n q} x_{n}$ one has $q$ divides each $y_{n}$, so each $y_{n} \cdot \alpha \in \mathbb{N}$. We claim $\operatorname{FS}\left(\left\langle y_{n} \cdot \alpha\right\rangle_{n=1}^{\infty}\right) \subseteq\{[n \alpha]: n \in A\}$. Let $F$ be a finite nonempty subset of $\mathbb{N}$. Then $\sum_{n \in F} y_{n} \cdot \alpha=\left[\left(\sum_{n \in F} y_{n}\right) \cdot \alpha\right]$ and $\sum_{n \in F} y_{n} \in A$.

2.5. Theorem. Let $\mathcal{B} \subseteq P(\mathbb{N})($ where $P(\mathbb{N})=\{A: A \subseteq \mathbb{N}\})$ and assume

(1) $\mathcal{B}$ is partition regular;

(2) whenever $\alpha>0,0<\gamma<1$, and $A \in \mathcal{B}$, one has $\{[n \alpha+\gamma]: n \in A\} \in \mathcal{B}$; and

(3) for each $A \in \mathcal{B}$ there exists $x, y \in A$ with $x+y \in A$. 
Then whenever $\alpha>0,0<\gamma<1$, and $A \in \mathcal{B}^{*}$ one has $\{[n \alpha+\gamma]: n \in A\} \in B^{*}$.

Proof. Let $\alpha<0,0<\gamma<1$, and $A \in B^{*}$ be given. Let $B=\{[n \alpha+\gamma]: n \in A\}$. Let $C \in \mathcal{B}$. We need to show that $C \cap B \neq \emptyset$. Let $\varepsilon=\min \{1 / 2, \gamma / \alpha,(1-\gamma) / \alpha\}$. By Lemma 2.2 (with $1 / \alpha$ replacing $\alpha$ ) pick $D \in B$ with $D \subseteq C$ and $f_{1 / \alpha}(k) \in(-\varepsilon, \varepsilon)$ whenever $k \in D$. Let $E=\{[k / \alpha+\varepsilon]: k \in D\}$. Then by hypothesis (2) with $1 / \alpha$ replacing $\alpha$ and $\varepsilon$ replacing $\gamma$ we have $E \in \mathcal{B}$. Therefore $E \cap A \neq \emptyset$ so pick $n \in E \cap A$ and pick $k \in D$ such that $n=[k / \alpha+\varepsilon]$. We show that $k=[n \alpha+\gamma]$ so that $k \in B \cap C$ as required. Let $l=h_{1 / \alpha}(k)$. Since $k \in D$ we have $l-\varepsilon<k / \alpha<l+\varepsilon$. Also $n \leq k / \alpha+\varepsilon<n+1$ so $n \leq k / \alpha+\varepsilon<l+2 \varepsilon \leq l+1$ so $n \geq l$. Also $l<k / \alpha+\varepsilon<n+1$ so $l \leq n$ and hence $l=n$. Therefore $n-\varepsilon<k / \alpha<n+\varepsilon$ so $n \alpha-1+\gamma \leq n \alpha-\varepsilon \alpha<k<n \alpha+\varepsilon \alpha \leq n \alpha+\gamma$ and hence $k<n \alpha+\gamma<k+1$ as required.

As a consequence of the following corollary we see for example that if $\alpha_{1}, \alpha_{2}>0$, and $0<\gamma_{1}<1$, and $0<\gamma_{2}<1$, then $\left\{\left[\left[n \alpha_{1}+\gamma_{1}\right] \alpha_{2}+\gamma_{2}\right]: n \in \mathbb{N}\right\}$ is a $\Delta^{*}$ set.

2.6. Corollary. Let $\alpha>0$, let $0<\gamma<1$, and let $A \subseteq \mathbb{N}$.

(a) If $A$ is a $\Delta^{*}$ set, then $\{[n \alpha+\gamma]: n \in A\}$ is a $\Delta^{*}$ set.

(b) If $A$ is an IP* set, then $\{[n \alpha+\gamma]: n \in A\}$ is an IP* set.

Proof. Theorems 2.3 and 2.5.

Again we see that with $\alpha$ rational one can allow $\gamma=0$. (See Theorem 6.3 for a proof that one cannot allow $\gamma=0$ with $\alpha$ irrational.)

2.7. Theorem. Let $\mathcal{B} \subseteq P(\mathbb{N})$ and assume

(1) for each $A \in \mathcal{B}$ and each $n \in \mathbb{N}, A \cap \mathbb{N} n \in \mathcal{B}$ and

(2) for each $\alpha>0$ with $\alpha \in \mathbb{Q}$ and each $A \in \mathcal{B}$ one has $\{[n \alpha]: n \in A\} \in \mathcal{B}$.

Then for each $\alpha>0$ with $\alpha \in \mathbb{Q}$ and each $A \in \mathcal{B}^{*}$ one has $\{[n \alpha]: n \in A\} \in \mathcal{B}^{*}$.

Proof. Let $\alpha>0$ with $\alpha \in \mathbb{Q}$ and $A \in \mathcal{B}^{*}$ be given. Pick $p, q \in \mathbb{N}$ with $\alpha=p / q$. Let $B=\{[n \alpha]: n \in A\}$ and let $C \in \mathcal{B}$. We show $B \cap C \neq \emptyset$. Now $C \cap \mathbb{N} p \in \mathcal{B}$ by assumption (1) so $D=\{[k / \alpha]: k \in C \cap \mathbb{N} p\} \in \mathcal{B}$ by assumption (2). Pick $n \in D \cap A$ and pick $k \in C \cap \mathbb{N} p$ such that $n=[k / \alpha]$. Since $p$ divides $k$ we have $n=k / \alpha$ so $k=n \alpha=[n \alpha]$ so $k \in B \cap C$.

2.8. Corollary. Let $\alpha>0$ with $\alpha \in \mathbb{Q}$ and let $A \subseteq \mathbb{N}$.

(a) If $A$ is a $\Delta^{*}$ set, then $\{[n \alpha]: n \in A\}$ is a $\Delta^{*}$ set.

(b) If $A$ is an IP* set, then $\{[n \alpha]: n \in A\}$ is an IP* set.

Proof. By Theorem 2.4 the classes $\Delta$ and $I P$ satisfy hypothesis (2) of Theorem 2.7. As in the proof of Theorem 2.4 one sees that the classes $\Delta$ and $I P$ satisfy hypothesis (1) of Theorem 2.7.

\section{DyNAMICAL PRELIMINARIES}

In this section we collect some definitions and results from topological dynamics which are pertinent to our presentation.

Given a compact metric space $(X, d)$ and a continuous $T: X \rightarrow X$, a point $y$ of $X$ is uniformly recurrent for $(X, T)$ if and only if whenever $U$ is a neighborhood of $y$, the set $\left\{n \in \mathbb{N}: T^{n}(y) \in U\right\}$ is syndetic, that is, has bounded gaps. Also points $x$ and $y$ are proximal if and only if for each $\varepsilon>0$ there is some $n \in \mathbb{N}$ with $d\left(T^{n}(x), T^{n}(y)\right)<\varepsilon$.

The notion of "central" was introduced by Furstenberg [12]. 
3.1. Definition. Let $A \subseteq \mathbb{N}$. We say $A$ is central via $(X, T, x, y, U)$ to mean that $X$ is a compact metric space, $T$ is a continuous function from $X$ to $X, x$ and $y$ are proximal in $X, y$ is uniformly recurrent, $U$ is a neighborhood of $y$ and $A=\left\{n \in \mathbb{N}: T^{n} x \in U\right\}$. A subset $A$ of $\mathbb{N}$ is central if and only if there exist $X, T, x, y$, and $U$ such that $A$ is central via $(X, T, x, y, U)$.

We now show that if $A$ is central via $(X, T, x, y, U)$ one may assume that $T$ is onto $X$.

3.2. Lemma. Let $A \subseteq \mathbb{N}$ be central. There exist $X, T, x, y$, and $U$ such that $T$ maps $X$ onto $X$ and $A$ is central via $(X, T, x, y, U)$.

Proof. Pick $Y, S, a, b$, and $V$ such that $A$ is cental via $(Y, S, a, b, V)$. Let $Z=\{0\} \cup$ $\{1 / n: n \in \mathbb{N}\}$ with the ordinary Euclidean metric. Let $X=Z \times Y$, and for $c \in Y$ and $n \in \mathbb{N}$ define $T(0, c)=(0, c), T(1, c)=(1, S(c))$, and $T(1 /(n+1), c)=(1 / n, c)$. Let $x=(1, a), y=(1, b)$, and $U=\{1\} \times V$. Then $T$ maps $X$ onto $X$ and $A$ is central via $(X, T, x, y, U)$.

The following lemma is well known, and we omit its proof.

3.3. Lemma. Let $(Y, d)$ be a compact metric space and let $X=Y^{\mathbb{Z}}$, the set of functions from $\mathbb{Z}$ to $Y$. For $f, g \in X$ define

$$
D(f, g)=\sup \{(1 /(|n|+1)) \cdot d(f(n), g(n)): n \in \mathbb{Z}\} .
$$

Then $D$ is a metric on $X$ and the metric and product topologies agree.

The proof of the following theorem is sketched in [12, pages 169, 170]. We strengthen the conclusion of Lemma 3.2 to obtain a homeomorphism.

3.4. Theorem. Let $A \subseteq \mathbb{N}$ be central. There exist $X, T, x, y$, and $U$ such that $T$ is a homeomorphism from $X$ onto $X$ and $A$ is central via $(X, T, x, y, U)$.

Proof. Pick, by Lemma 3.2, $Y, S, a, b$, and $V$ such that $S$ maps $Y$ onto $Y$ and $A$ is central via $(Y, S, a, b, V)$. Let $X=\left\{f \in Y^{\mathbb{Z}}\right.$ : for all $\left.n \in \mathbb{Z}, f(n+1)=S(f(n))\right\}$. Then $X$ is closed in $Y^{\mathbb{Z}}$. Indeed if $f \in Y^{\mathbb{Z}} \backslash X$ pick $n \in \mathbb{Z}$ and disjoint neighborhoods $U_{1}$ and $W$ of $f(n+1)$ and $S(f(n))$ respectively. Pick a neighborhood $U_{2}$ of $f(n)$ with $S\left[U_{2}\right] \subseteq W$. Then $\pi_{n+1}^{-1}\left[U_{1}\right] \cap \pi_{n}^{-1}\left[U_{2}\right]$ is a neighborhood of $f$ missing $X$. Thus by Lemma $3.3, X$ is a (compact) metric space.

Define $T: X \rightarrow X$ as the restriction of the shift. That is, $T(f)(n)=f(n+1)$. Then $T$ is easily seen to be a homeomorphism of $X$ onto $X$.

Now we observe that

$(*)$ if $n \in \mathbb{N}, i \in \mathbb{Z}, n+i \geq 0$, and $f \in X$, then $T^{n}(f)(i)=S^{n+i}(f(0))$.

This fact is easily established by induction on $(n+i)$.

We also observe that

$(* *)$ if $c \in Y$ then $\pi_{0}^{-1}[\{c\}] \cap X \neq \emptyset$. (That is, there exists $f \in X$ with $f(0)=c$.) To see this for $n \geq 0$ let $f(n)=S^{n}(c)$ and inductively for $n \leq 0$ pick $f(n-1)$ such that $S(f(n-1))=f(n)$, which one can do since $S$ is onto $Y$.

Now let $W=\operatorname{cl}_{Y}\left\{S^{n}(b): n \in \mathbb{N}\right\}$. Since $b$ is uniformly recurrent, $W$ is minimal closed $S$ invariant [12, Theorem 1.17]. Then $\pi_{0}^{-1}[W] \cap X$ is closed and $T$ invariant so (by Zorn's Lemma) pick $Z \subseteq \pi_{0}^{-1}[W] \cap X$ which is minimal closed $T$ invariant. Then $\pi_{0}[Z]$ is a closed $S$ invariant subset of $W$ and hence $\pi_{0}[Z]=W$. Pick $y \in Z$ such that $\pi_{0}(y)=b$. Since $Z$ is minimal closed $T$ invariant, $y$ is uniformly recurrent in $(X, T)$ [12, Theorem 1.15]. 
By $(* *)$ pick some $x \in X$ such that $\pi_{0}(x)=a$. We show now that $x$ and $y$ are proximal. First observe that since $Y$ is compact, $S$ is uniformly continuous so for each $\varepsilon>0$ pick $h(\varepsilon)>0$ (with $h(\varepsilon) \leq \varepsilon$ ) so that for all $u, v \in Y$, if $d(u, v)<h(\varepsilon)$, then $d(S(u), S(v))<\varepsilon$. Now let $\varepsilon>0$ be given. We need to produce some $n \in \mathbb{N}$ such that $D\left(T^{n} x, T^{n} y\right)<\varepsilon$, where $D$ is as in Lemma 3.3. Let $M=\sup \{d(u, v): u, v \in Y\}$ and pick $k \in \mathbb{N}$ such that $1 /(k+1)<\varepsilon /(2 M)$. Let $\delta=h^{2 k}(\varepsilon / 2)$ and pick $m \in \mathbb{N}$ such that $d\left(S^{m}(a), S^{m}(b)\right)<\delta$. Then given $t \in\{0,1, \ldots, 2 k\}$ we have $d\left(S^{m+t}(a), S^{m+t}(b)\right)<h^{2 k-t}(\varepsilon / 2) \leq \varepsilon / 2$. Let $n=m+k$. Then one easily verifies that $D\left(T^{n} x, T^{n} y\right) \leq \varepsilon / 2<\varepsilon$.

Finally let $U=\pi_{0}^{-1}[V]$. Then using $(*)$ we have for $n \in \mathbb{N}$ that

$$
\begin{aligned}
T^{n}(x) \in U & \Leftrightarrow T^{n}(x)(0) \in V \\
& \Leftrightarrow S^{n}(x(0)) \in V \\
& \Leftrightarrow n \in A .
\end{aligned}
$$

3.5. Lemma. Let $X$ be a compact metric space, let $T$ be a homeomorphism from $X$ onto $X$, and let $y \in X$. If $y$ is uniformly recurrent for $(X, T)$, then it is uniformly recurrent for $\left(X, T^{-1}\right)$.

Proof. Let $W=\operatorname{cl}\left\{T^{n} y: n \in \mathbb{N}\right\}$. Since $y$ is uniformly recurrent, $W$ is minimal closed $T$ invariant. But then $W$ is $T^{-1}$ invariant. (To see this let $x \in W$ and suppose $T^{-1} x \notin W$. Pick an open neighborhood $U$ of $T^{-1} x \operatorname{missing} W$. Then $W \backslash T[U]$ is a proper closed subset of $W$ so some $z \in W \backslash T[U]$ has $T z \notin W \backslash T[U]$. Then $T z \in T[U]$, so $z \in U \cap W$, a contradiction.) Pick a minimal closed $T^{-1}$ invariant subset $A$ of $W$. By the above argument $A$ is $T$ invariant so $A=W$. Since $y$ is a member of a minimal closed $T^{-1}$ invariant set, $y$ is uniformly recurrent for $\left(X, T^{-1}\right)$.

We omit the proof of the following well known result.

3.6. Lemma. Let $X$ be a compact metric space and let $T$ be a homeomorphism from $X$ to $X$. In $X \times[0,1]$ identify $(x, 1)$ and $(T x, 0)$ for all $x \in X$ and let $Y=X \times[0,1)$ have the quotient topology resulting from this identification. Then $Y$ is a compact metrizable space and for each $s \in(0,1)$ the function $F_{s}: Y \rightarrow Y$ defined by $F_{s}(x, t)=\left(T^{[s+t]} x, s+t-[s+t]\right)$ is continuous.

3.7. Lemma. Let $X, T, Y$ and $F_{s}($ for $0<s<1)$ be as in Lemma 3.6 Let $y \in X$. If $y$ is uniformly recurrent for $(X, T)$, then there exists $\tau \in(0,1)$ such that $(y, \tau)$ is uniformly recurrent for $\left(Y, F_{s}\right)$.

Proof. Let $W=\operatorname{cl}_{X}\left\{T^{n} y: n \in \mathbb{N}\right\}$. Since $y$ is uniformly recurrent, $W$ is minimal closed $T$ invariant. Then $W \times[0,1)$ is a closed $F_{s}$ invariant subset of $Y$. (To see that $W \times[0,1)$ is closed observe that if $x \in Y \backslash W$ and $U$ is a neighborhood of $x$ missing $W$, then $T^{-1} U$ also misses $W$ so that $(U \times[0,1)) \cup\left(T^{-1}[U] \times[0,1)\right)$ is a neighborhood of $(x, 0)$ missing $W \times[0,1)$.)

Pick $Z$, a minimal closed $F_{s}$ invariant subset of $W \times[0,1)$. Consider $P: Y \rightarrow X$, the projection onto the first coordinate. (Be cautioned that $Y$ does not have the product topology so $P$ need not be continuous.)

We claim first $P[Z]$ is $T$ invariant. Indeed, given $(x, t) \in Z$ pick the least positive integer $n$ such that $t+s n>1$. Then $F_{s}^{n}(x, t)=(T x, t+s n-1) \in Z$. 
Now we claim $P[Z]$ is closed. Let $x \in X \backslash P[Z]$. For each $t \in(0,1)$ pick $\varepsilon_{t}>0$ with $\varepsilon_{t} \leq \min (t, 1-t)$ and pick $U_{t}$ a neighborhood of $x$ such that $\left(U_{t} \times V_{t}\right) \cap Z=\emptyset$ where $V_{t}=\left(t-\varepsilon_{t}, t+\varepsilon_{t}\right)$. Likewise pick $U_{0}$ a neighborhood of $x$ and $\varepsilon_{0}>0$ with $\left(U_{0} \times V_{0}\right) \cap Z=\emptyset$ where $V_{0}=\left[0, \varepsilon_{0}\right)$. Now $F_{s}[Z]$ is closed and $F_{s}$ invariant so $F_{s}[Z]=Z$. Thus if we had $(T x, 0) \in Z$ we would have $(x, 1-s) \in Z$ and hence $x \in P[Z]$. Therefore $(T x, 0) \notin Z$ so we may pick a neighborhood $U_{1}$ of $x$ and $\varepsilon_{1}>0$ with $\left(U_{1} \times V_{1}\right) \cap Z=\emptyset$ where $V_{1}=\left(1-\varepsilon_{1}, 1\right)$. Pick finite $F \subseteq[0,1]$ with $[0,1) \subseteq \bigcup_{t \in F} V_{t}$. Let $U=\bigcap_{t \in F} U_{t}$. Then $U \cap P[Z]=\emptyset$ as required.

Now, $P[Z]$ is a closed $T$ invariant subset of $W$ so $P[Z]=W$ so we may pick $t \in[0,1)$ with $(y, t) \in Z$. If $t>0$, let $\tau=t$. If $t=0$, let $\tau=t+s$ so that $(y, \tau)=F_{s}(y, t)$. In either case $(y, \tau) \in Z$ and is hence uniformly recurrent for $\left(Y, F_{s}\right)$.

3.8. Lemma. Let $A \subseteq \mathbb{N}$ be a central and let $n \in \mathbb{N}$.

(a) $A / n=\{m \in \mathbb{N}: m n \in A\}$ is central.

(b) An is central.

Proof. (a) This is [12, Lemma 8.24]. (If $A$ is central, via $T, A / n$ is central via $T^{n}$.)

(b) Pick $Y, S, a, b$, and $V$ such that $A$ is central via $(Y, S, a, b, V)$. Let $X=$ $Y \times\{1,2,3, \ldots, n\}$, where $\{1,2,3, \ldots, n\}$ is discrete, and define $T: X \rightarrow X$ by $T(y, k)=(y, k+1)$ for $k<n$ and $T(y, n)=(S y, 1)$. Then $T^{k n}(y, 1)=\left(S^{k} y, 1\right)$ for all $k \in \mathbb{N}$. Let $x=(a, 1), y=(b, 1)$, and $U=V \times\{1\}$. Then $A n$ is central via $(X, T, x, y, U)$.

\section{Dynamical Results}

We begin by establishing the analogue of Theorem 2.3 for central sets.

4.1. Theorem. Let $\alpha>0$, let $0<\gamma<1$ and let $A \subseteq \mathbb{N}$. If $A$ is central, then $\{[n \alpha+\gamma]: n \in A\}$ is central.

Proof. Let $\delta=\min \{\gamma, 1-\gamma\}$ and let $B=\{k \in \mathbb{N}$ : for some $n \in A,|k-n \alpha|<\delta\}$. Then $B \subseteq\{[n \alpha+\gamma]: n \in A\}$. Indeed, if $|k-n \alpha|<\delta$, then $\gamma-1<k-n \alpha<\gamma$ so $k=[n \alpha+\gamma]$. Thus it suffices to show that $B$ contains a central set. Pick $m \in \mathbb{N}$ such that $1 /(m \alpha)<1$ and let $s=1 /(m \alpha)$. Let $\mu=\min \{s, 1-s\}$. Now by Lemma $3.8(\mathrm{a}), A / m=\{n \in \mathbb{N}: n m \in A\}$ contains a central set. Pick by Theorem 3.4 a compact metric space $X$, a homeomorphism $T$ of $X$ onto $X$, a uniformly recurrent point $y$ of $X$, a point $x$ proximal to $y$, and a neighborhood $U$ of $y$ such that $\left\{n \in \mathbb{N}: T^{n} x \in U\right\} \subseteq A / m$. Let $Y$ and $F_{s}$ be as in Lemma 3.6 and pick by Lemma 3.7 some $\tau \in(0,1)$ such that $(y, \tau)$ is uniformly recurrent for $\left(Y, F_{s}\right)$.

We now claim that $(x, \tau)$ and $(y, \tau)$ are proximal. Choose a sequence $\langle n(k)\rangle_{k=1}^{\infty}$ in $\mathbb{N}$ such that $\lim _{k \rightarrow \infty} d\left(T^{n(k)} x, T^{n(k)} y\right)=0$. Passing to a subsequence we may presume we have $a \in X$ such that $a=\lim _{k \rightarrow \infty} T^{n(k)} y=\lim _{k \rightarrow \infty} T^{n(k)} x$. For each $k \in \mathbb{N}$ choose $r(k) \in \mathbb{N}$ such that $[s \cdot r(k)+\tau]=n(k)$ and $n(k)+\mu / 2 \leq s \cdot r(k)+\tau<$ $n(k)+1-\mu / 2$, and let $t(k)=s \cdot r(k)+\tau-n(k)$. Then $F_{s}^{r(k)}(x, \tau)=\left(T^{n(k)} x, t(k)\right)$ and $F_{s}^{r(k)}(y, \tau)=\left(T^{n(k)} y, t(k)\right)$. Let $l$ be a cluster point of $\langle t(k)\rangle_{k=1}^{\infty}$ and note $l \in[\mu / 2,1-\mu / 2]$. Let $b=(a, l)$. Then given any neighborhood $W$ of $b$ one has some $k$ with $F_{s}^{r(k)}(x, \tau) \in W$ and $F_{s}^{r(k)}(y, \tau) \in W$ and hence $(x, \tau)$ and $(y, \tau)$ are proximal as claimed.

Now let $\varepsilon=\min \{\tau, 1-\tau, \delta / m \alpha\}$ and let $V=U \times(\tau-\varepsilon, \tau+\varepsilon)$. Then $V$ is a neighborhood of $(y, \tau)$. Let $C=\left\{k \in \mathbb{N}: F_{s}^{k}(x, \tau) \in V\right\}$. Then $C$ is central. 
We claim $C \subseteq B$. To this end let $k \in C$. Let $n=[k s+\tau]=[k /(m \alpha)+\tau]$. Then $\left(T^{n} x, k /(m \alpha)+\tau-n\right)=F_{s}^{k}(x, \tau) \in V$. Since $T^{n} x \in U$ we have $n \in A / m$ so $n m \in A$. Also $\tau-\varepsilon<k /(m \alpha)+\tau-n<\tau+\varepsilon$ so $-\delta \leq-\varepsilon m \alpha<k-n m \alpha<\varepsilon m \alpha \leq \delta$ so $|k-n m \alpha|<\delta$ and hence $k \in B$.

4.2. Lemma. Let $\alpha>0$ with $\alpha \in \mathbb{Q}$ and let $A \subseteq \mathbb{N}$. If $A$ is a central set, then $\{[n \alpha]: n \in A\}$ is a central set.

Proof. Let $\alpha=p / q$ with $p, q \in \mathbb{N}$. By Lemma 3.8(b) $A p$ is central and hence by Lemma 3.8(a) $(A p) / q$ is central and $(A p) / q \subseteq\{[n \alpha]: n \in A\}$.

4.3. Theorem. Let $\alpha>0$ and let $0 \leq \gamma<1$ with $\gamma>0$ if $\alpha \notin \mathbb{Q}$ and let $A \subseteq \mathbb{N}$. If $A$ is central ${ }^{*}$, then $\{[n \alpha+y]: n \in A\}$ is central* .

Proof. For the case $\gamma>0$ let $\mathcal{B}=\{B \subseteq \mathbb{N}: B$ contains a central set $\}$. (We know algebraically that $\mathcal{B}$ is the class of central sets.) In any event $A \in B^{*}$ if and only if $A$ is a central ${ }^{*}$ set so Theorems 2.5 and 4.1 together with the fact from [5, Corollary $6.12]$ that central sets are partition regular yield the desired conclusion.

For the case $\gamma=0$ and $\alpha \in \mathbb{Q}$ we can apply Theorem 2.7 and Lemma 4.2 once we have shown that given a central set $A$ and $n \in \mathbb{N}$ one has that $A \cap \mathbb{N} n$ is central. Since $A \cap \mathbb{N} n=(A / n) n$, this follows from Lemma 3.8.

The following result is of independent interest because at least from the point of view of the algebraic characterization of central it is quite surprising. (From that point of view one finds more natural the fact that $\{m \in \mathbb{N}: A-m$ is central $\}$ is central, which follows immediately from the algebraic definition.)

4.4. Theorem. Let $A \subseteq \mathbb{N}$ be central and let $B=\{m \in \mathbb{N}: A+m$ is central $\}$. Then $B$ is a central set.

Proof. Pick by Theorem $3.4 X, T, x, y$, and $U$ such that $T$ is a homeomorphism from $X$ onto $X$ and $A$ is central via $(X, T, x, y, U)$. By Lemma $3.5 y$ is uniformly recurrent for $T^{-1}$ so $C=\left\{m \in \mathbb{N}: T^{-m} y \in U\right\}$ is central via $\left(X, T^{-1}, y, y, U\right)$. We show that $C \subseteq B$. Let $m \in C$. Then $y \in T^{m}[U]$ which is open since $T$ is a homeomorphism. Then $A+m=\left\{n \in \mathbb{N}: T^{n} x \in T^{m}[U]\right\}$ so $A+m$ is central.

\section{Algebraic preliminaries}

In [5] it was shown that the dynamical definition of "central" given in Section 3 is equivalent to the following simple algebraic characterization: A subset $A$ of $\mathbb{N}$ is central if and only if $A$ is a member of some minimal idempotent of $(\beta \mathbb{N},+)$ where an idempotent is minimal if and only if it is a member of some minimal right ideal. (Equivalently an idempotent is minimal if and only if it is minimal with respect to the ordering of idempotents wherein $p \leq q$ if and only if $p=p+q=q+p$. See $[5$, Lemma 3.2].) (One can also verify that an idempotent $p$ is minimal if and only if the dynamical system $(X, T)$ is minimal where $X=p+\beta \mathbb{N}$ and $T(q)=q+1$.)

From this characterization of central it is immediate that a set $A$ is central* if and only if $A$ is a member of every minimal idempotent.

It is a well known result of Galvin (see [20, Theorem 2.3(b) and Theorem 2.5]) that a set $A$ is an $I P$ set if and only if $A$ is a member of some idempotent of $(\beta \mathbb{N},+)$. Consequently $A$ is an $I P^{*}$ set if and only if $A$ is a member of every idempotent.

We now establish an algebraic characterization of $\Delta$ sets and $\Delta^{*}$ sets. We expand our horizons temporarily to work with the semigroup $(\beta \mathbb{Z},+)$. Strictly speaking $\beta \mathbb{N}$ 
is not a subset of $\beta \mathbb{Z}$ since an ultrafilter on $\mathbb{N}$ is not an ultrafilter on $\mathbb{Z}$. However, given $p \in \beta \mathbb{N}$, the family $\{A \subseteq \mathbb{Z}: A \cap \mathbb{N} \in p\}$ is an ultrafilter on $\mathbb{Z}$, so we may reasonably pretend that $\beta \mathbb{N} \subseteq \beta \mathbb{Z}$ (just as we pretend all along that $\mathbb{N} \subseteq \beta \mathbb{N}$ ).

Given $p \in \beta \mathbb{N},-p=\{A \subseteq \mathbb{Z}:-A \cap \mathbb{N} \in p\}$ is an ultrafilter on $\mathbb{Z}$. One should be cautioned that $p-p=p+(-p) \neq 0$ unless $p \in \mathbb{N}$. Since $\beta \mathbb{N} \backslash \mathbb{N}$ is a right ideal of $(\beta \mathbb{Z},+$ ), one has $p-p \in \beta \mathbb{N} \backslash \mathbb{N}$ whenever $p \in \beta \mathbb{N} \backslash \mathbb{N}$. (However $(-p)+p \notin \beta \mathbb{N}$; it is in $-\beta \mathbb{N}$.)

5.1. Theorem. Let $A \subseteq \mathbb{N}$.

(a) $A$ is a $\Delta$ set if and only if there exists $p \in \beta \mathbb{N} \backslash \mathbb{N}$ such that $A \in p-p$.

(b) $A$ is a $\Delta^{*}$ set if and only if for every $p \in \beta \mathbb{N} \backslash \mathbb{N}, A \in p-p$.

Proof. (a) Assume $A$ is a $\Delta$ set and pick an infinite increasing sequence $\left\langle x_{n}\right\rangle_{n=1}^{\infty}$ in $\mathbb{N}$ with $\left\{x_{m}-x_{n}: n<m\right\} \subseteq A$. Pick $p \in \beta \mathbb{N} \backslash \mathbb{N}$ with $\left\{x_{n}: n \in \mathbb{N}\right\} \in p$. To see that $A \in p-p$ we show $\left\{-x_{n}: n \in \mathbb{N}\right\} \subseteq\{y \in \mathbb{Z}: A-y \in p\}$. To this end let $n \in \mathbb{N}$ be given. Then since $p$ is non-principal, $\left\{x_{m}: m>n\right\} \in p$ and $\left\{x_{m}: m>n\right\} \subseteq A-\left(-x_{n}\right)$, so $A-\left(-x_{n}\right) \in p$ as required.

Now assume we have some $p \in \beta \mathbb{N} \backslash \mathbb{N}$ with $A \in p-p$. Let $B=\{x \in \mathbb{Z}: A-x \in p\}$. Then $B \in-p$ so $(-B) \cap \mathbb{N} \in p$. Let $C_{1}=A \cap(-B)$ and pick $x_{1} \in C_{1}$. Then $-x_{1} \in B$ so $A+x_{1} \in p$. Inductively let $C_{n+1}=C_{n} \cap(-B) \cap\left(A+x_{n}\right)$. Then $C_{n+1} \in p$ so pick $x_{n+1} \in C_{n+1}$ with $x_{n+1}>x_{n}$. Then given $m>n$ we have $x_{m} \in C_{m} \subseteq C_{n+1} \subseteq A+x_{n}$ so $x_{m}-x_{n} \in A$.

(b) Given that $A$ is a $\Delta^{*}$ set one can't have $\mathbb{N} \backslash A \in p-p$ for any $p \in \beta \mathbb{N} \backslash \mathbb{N}$ since $A \cap(\mathbb{N} \backslash A)=\emptyset$ so $A \in p-p$. Likewise given an $A \in p-p$ for all $p \in \beta \mathbb{N} \backslash \mathbb{N}$ and given a $\Delta$ set $B$ we have $B \in p+(-p)$ for some $p \in \beta \mathbb{N} \backslash \mathbb{N}$ so $A \cap B \neq \emptyset$.

Recall that for $\alpha>0$ we have defined $f_{\alpha}: \mathbb{N} \rightarrow T$ and $h_{\alpha}: \mathbb{N} \rightarrow \mathbb{N}$ in Definition 2.1. One has $h_{\alpha}(n)$ is the nearest integer to $n \alpha$ and $f_{\alpha}(n)$ is the fractional part of $n \alpha$.

5.2. Definition. Let $\alpha>0, \gamma>0$. Define $g_{\alpha, \gamma}: \mathbb{N} \rightarrow \mathbb{N} \cup\{0\}$ by $g_{\alpha, \gamma}(n)=[n \alpha+\gamma]$. (Then $h_{\alpha}=g_{\alpha, 1 / 2}$.)

Since $T$ is compact, $f_{\alpha}$ has a continuous extension to $\beta \mathbb{N}$ which we also denote by $f_{\alpha}$. Likewise $g_{\alpha, \gamma}: \mathbb{N} \rightarrow \mathbb{N} \cup\{0\} \subseteq \beta(\mathbb{N} \cup\{0\})=\beta \mathbb{N} \cup\{0\}$ so $g_{\alpha, \gamma}$ has a continuous extension to $\beta \mathbb{N}$ which we also denote by $g_{\alpha, \gamma}$. Similarly, we denote the continuous extension of $h_{\alpha}$ again by $h_{\alpha}$.

It is easy to see that, since $f_{\alpha}$ is a homomorphism on $(\mathbb{N},+)$, the extension to $(\beta \mathbb{N},+)$ is also a homomorphism. On the other hand, $g_{\alpha, \gamma}$ is usually not a homomorphism. We shall see however, in Theorem 5.10 below, that the restriction to certain special subsets is often a homomorphism.

For points $x, y$, and $z$ in $T$, when we write $x<y<z$ we mean $y$ is on the counterclockwise arc from $x$ to $z$.

5.3. Definition. Let $\alpha \in \mathbb{R}$ with $\alpha>0$.

(a) $U_{\alpha}=\left\{p \in \beta \mathbb{N}:\left\{n \in \mathbb{N}: f_{\alpha}(p)<f_{\alpha}(n)<f_{\alpha}(p)+1 / 2\right\} \in p\right\}$.

(b) $D_{\alpha}=\left\{p \in \beta \mathbb{N}:\left\{n \in \mathbb{N}: f_{\alpha}(p)-1 / 2<f_{\alpha}(n)<f_{\alpha}(p)\right\} \in p\right\}$.

(c) $Z_{\alpha}=\left\{p \in \beta \mathbb{N}: f_{\alpha}(p)=0\right\}$.

(d) $X_{\alpha}=U_{\alpha} \cap Z_{\alpha}$.

(e) $Y_{\alpha}=D_{\alpha} \cap Z_{\alpha}$.

The sets $U_{\alpha}$ and $D_{\alpha}$ consist of those points of $\beta \mathbb{N}$ for which $f_{\alpha}$ approaches its value from above and from below respectively. We shall see in Theorems 5.5 and 5.6 below that these are interesting algebraic objects. 
5.4. Lemma. If $\alpha>0$ is irrational, then $U_{\alpha} \cup D_{\alpha}=\beta \mathbb{N} \backslash \mathbb{N}$ and for each $\varepsilon>0$ and each $p \in U_{\alpha}$ and each $q \in D_{\alpha},\left\{n \in \mathbb{N}: f_{\alpha}(p)<f_{\alpha}(n)<f_{\alpha}(p)+\varepsilon\right\} \in p$ and $\left\{n \in \mathbb{N}: f_{\alpha}(q)-\varepsilon<f_{\alpha}(n)<f_{\alpha}(q)\right\} \in q$. If $\alpha>0$ is rational, then $U_{\alpha} \cup D_{\alpha}=\emptyset$.

Proof. First assume $\alpha$ is irrational. Given $n \in \mathbb{N},\left\{m \in \mathbb{N}: f_{\alpha}(m)=f_{\alpha}(n)\right\}$ is a member of the principal ultrafilter generated by $n$ (which you will recall we have identified with $n$ ). Thus $U_{\alpha} \cup D_{\alpha} \subseteq \beta \mathbb{N} \backslash \mathbb{N}$. Now given $p \in \beta \mathbb{N} \backslash \mathbb{N}$ we have $\left|\left\{n \in \mathbb{N}: f_{\alpha}(n)=f_{\alpha}(p)\right\}\right| \leq 1$ so either $\left\{n \in \mathbb{N}: f_{\alpha}(p)-1 / 2<f_{\alpha}(n)<f_{\alpha}(p)\right\} \in p$ or $\left\{n \in \mathbb{N}: f_{\alpha}(p)<f_{\alpha}(n)<f_{\alpha}(p)+1 / 2\right\} \in p$.

Now given $\varepsilon>0$ (which we may presume is less than $1 / 2$ ), $p \in U_{\alpha}$, and $q \in D_{\alpha}$ observe that, by the continuity of $f_{\alpha},\left\{n \in \mathbb{N}: f_{\alpha}(p)-\varepsilon<f_{\alpha}(n)<f_{\alpha}(p)+\varepsilon\right\} \in p$ and $\left\{n \in \mathbb{N}: f_{\alpha}(q)-\varepsilon<f_{\alpha}(n)<f_{\alpha}(q)+\varepsilon\right\} \in q$ so the conclusion follows.

Now, if $\alpha$ is rational one has $\left\{f_{\alpha}(n): n \in \mathbb{N}\right\}$ is finite so for any $p \in \beta \mathbb{N}$, $\left\{n \in \mathbb{N}: f_{\alpha}(n)=f_{\alpha}(p)\right\} \in p$.

The following result (done explicitly in the case $\alpha=1 /(2 \pi)$ with essentially the same proof which we use) is due to Baker and Milnes [2]. Recall that for $a, b, c \in \mathbb{T}$ we write $a<b<c$ to mean that $b$ is on the counterclockwise $\operatorname{arc}$ from $a$ to $c$. It is an exercise to verify that if $a<b<c, d<e<f, 0<c-a \leq 1 / 2,0<f-d \leq 1 / 2$ and one of $c-a<1 / 2$ or $f-d<1 / 2$, then $a-f<b-e<c-d$.

5.5. Theorem. Let $\alpha>0$ be irrational. Then $U_{\alpha}$ and $D_{\alpha}$ are left ideals of $(\beta \mathbb{N},+)$.

Proof. We prove the statement for $U_{\alpha}$, the other case being nearly identical. Let $p \in U_{\alpha}$ and let $q \in \beta \mathbb{N}$. Let $x=f_{\alpha}(p), y=f_{\alpha}(q)$, and let $r=q+p$. Since $f_{\alpha}$ is a homomorphism we have $x+y=f_{\alpha}(r)$. Suppose $r \notin U_{\alpha}$. Since $\beta \mathbb{N} \backslash \mathbb{N}$ is an ideal of $(\beta \mathbb{N},+)$ we have $r \in \beta \mathbb{N} \backslash \mathbb{N}$ so by Lemma $5.4, r \in D_{\alpha}$. For each $k \in \mathbb{N}$ let $A_{k}=\left\{n \in \mathbb{N}: x<f_{\alpha}(n)<x+1 / k\right\}, B_{k}=\left\{n \in \mathbb{N}: x+y-1 / k<f_{\alpha}(n)<x+y\right\}$ and $C_{k}=\left\{n \in \mathbb{N}: B_{k}-n \in q\right\}$. Since $p \in U_{\alpha}$ we have each $A_{k} \in p$. Since $r \in D_{\alpha}$ we have each $B_{k} \in r$. Since $r=q+p$, we have each $C_{k} \in p$. Pick $n \in A_{2} \cap C_{2}$. Now $x<f_{\alpha}(n)<x+1 / 2$ so pick $k \in \mathbb{N}$ with $x+2 / k<f_{\alpha}(n)<x+1 / 2$. Pick $m \in A_{k} \cap C_{k}$. Now $x<f_{\alpha}(m)<x+1 / k$ so $1 / k<f_{\alpha}(n)-f_{\alpha}(m)<1 / 2$. Since $n \in C_{2}$ and $m \in C_{k}$ we have $\left(B_{2}-n\right) \cap\left(B_{k}-m\right) \in q$ so pick $t \in\left(B_{2}-n\right) \cap\left(B_{k}-m\right)$. Then $x+y-1 / 2<f_{\alpha}(t+n)<x+y$ and $x+y-1 / k<f_{\alpha}(t+m)<x+y$. Since $f_{\alpha}(t+n)=f_{\alpha}(t)+f_{\alpha}(n)$ and $f_{\alpha}(t+m)=f_{\alpha}(t)+f_{\alpha}(m)$ we conclude that $-1 / 2<f_{\alpha}(n)-f_{\alpha}(m)<1 / k$, a contradiction.

There is a similar multiplicative result.

5.6. Theorem. Let $\alpha>0$ be irrational. Then $X_{\alpha}$ and $Y_{\alpha}$ are right ideals of $(\beta \mathbb{N}, \cdot)$.

Proof. We establish the statement for $Y_{\alpha}$. Let $p \in Y_{\alpha}$ and let $q \in \beta \mathbb{N}$. To show that $p \cdot q \in Y_{\alpha}$ it suffices to show that, for every $\varepsilon>0,\left\{n \in \mathbb{N}:-\varepsilon<f_{\alpha}(n)<0\right\} \in p \cdot q$ (for this then forces $f_{\alpha}(p \cdot q)=0$ and $p \cdot q \in D_{\alpha}$ ). To this end let $\varepsilon>0$ be given (with $\varepsilon<1$ ) and let $A=\left\{n \in \mathbb{N}:-\varepsilon<f_{\alpha}(n)<0\right\}$. We show that, for all $n \in \mathbb{N}, A / n \in p$, and hence that $A \in p \cdot q$. To this end let $n \in \mathbb{N}$. Let $B=\left\{m \in \mathbb{N}:-\varepsilon / n<f_{\alpha}(m)<0\right\}$. Then $B \in p$ and so it suffices to show that $B \subseteq A / n$. Let $m \in B$ and let $k=[m \alpha]$. Then $k+1-\varepsilon / n<m \alpha<k+1$ so $k n+n-\varepsilon<n m \alpha<k n+n$ so $[n m \alpha]=k n+n-1$ and $-\varepsilon<f_{\alpha}(n m)<0$ so that $n m \in A$ as required. 
Observe that $f_{\alpha}$ is not a homomorphism on $(\mathbb{N}, \cdot)$. The above proof however utilizes the fact that it is nearly a homomorphism around 0 .

5.7. Lemma. For any $\alpha>0, Z_{\alpha}$ is a compact subsemigroup of $(\beta \mathbb{N},+)$. If $\alpha$ is irrational, $X_{\alpha}$ and $Y_{\alpha}$ are subsemigroups of $(\beta \mathbb{N},+)$. If $\alpha=m / n$ where $m$ and $n$ are relatively prime natural numbers, then $Z_{\alpha}=\overline{\mathbb{N} n}$.

Proof. For the first assertion observe that $Z_{\alpha}$ is the kernel of a continuous homomorphism. The second assertion follows from the first assertion and Theorem 5.5. For the last assertion observe that the range of $f_{\alpha}$ is finite and that for $k \in \mathbb{N}$, $f_{\alpha}(k)=0$ if and only if $k \in \mathbb{N} n$.

We see in the next two results that we are really only concerned with three functions from $\beta \mathbb{N}$ to $\beta \mathbb{N}$, namely $g_{\alpha, 0}, g_{\alpha, 1}$, and $h_{\alpha}$.

5.8. Theorem. Let $\alpha>0$, let $0<\gamma<1$ and let $p \in Z_{\alpha}$. Then $g_{\alpha, \gamma}(p)=h_{\alpha}(p)$. If $\alpha \in \mathbb{Q}$, then $g_{\alpha, 0}(p)=h_{\alpha}(p)$.

Proof. Let $\varepsilon=\min \{\gamma, 1-\gamma\}$ and let $B=\left\{n \in \mathbb{N}:-\varepsilon<f_{\alpha}(n)<\varepsilon\right\}$. Since $f_{\alpha}(p)=0$ we have $B \in p$. We show that $g_{\alpha, \gamma}$ and $h_{\alpha}$ agree on $B$. Let $m=h_{\alpha}(n)$. Then $m-\varepsilon<\alpha n<m+\varepsilon$ so $m \leq m-\varepsilon+\gamma<\alpha n+\gamma<m+\varepsilon+\gamma \leq m+1$ so $m=[\alpha n+\gamma]=g_{\alpha, \gamma}(n)$.

If $\alpha=m / n$ in lowest terms we have by Lemma 5.7 that $Z_{\alpha}=\overline{\mathbb{N} n}$. Since $g_{\alpha, 0}$ and $h_{\alpha}$ agree on $\mathbb{N} n$ we have $g_{\alpha, 0}(p)=h_{\alpha}(p)$.

5.9. Lemma. Let $\alpha>0$ be irrational and let $p \in X_{\alpha}$ and $q \in Y_{\alpha}$. Then $g_{\alpha, 0}(p)=$ $h_{\alpha}(p)$ and $g_{\alpha, 1}(q)=h_{\alpha}(q)$.

Proof. We establish the result for $q$. Let $B=\left\{n \in \mathbb{N}:-1 / 2<f_{\alpha}(n)<0\right\}$. Since $q \in Y_{\alpha}$ we have $B \in q$. We show $h_{\alpha}$ and $g_{\alpha, 1}$ agree on $B$. Let $n \in B$ and let $m=h_{\alpha}(n)$. Then $m-1 / 2<\alpha n<m$ so $m+1 / 2<\alpha n+1<m+1$ and therefore $m=[\alpha n+1]=g_{\alpha, 1}(n)$.

5.10. Theorem. Let $\alpha>0$. Then $h_{\alpha}$ is an isomorphism and a homeomorphism from $Z_{\alpha}$ onto $Z_{1 / \alpha}$ with inverse $h_{1 / \alpha}$. If $\alpha$ is irrational, $h_{\alpha}$ takes $X_{\alpha}$ onto $Y_{1 / \alpha}$ and takes $Y_{\alpha}$ onto $X_{1 / \alpha}$.

Proof. We show

(1) if $p \in Z_{\alpha}$, then $h_{\alpha}(p) \in Z_{1 / \alpha}$,

(2) if $p, q \in Z_{\alpha}$, then $h_{\alpha}(p+q)=h_{\alpha}(p)+h_{\alpha}(q)$,

(3) if $p \in Z_{\alpha}$, then $h_{1 / \alpha}\left(h_{\alpha}(p)\right)=p$,

(4) if $p \in X_{\alpha}$, then $h_{\alpha}(p) \in Y_{1 / \alpha}$, and

(5) if $p \in Y_{\alpha}$, then $h_{\alpha}(p) \in X_{1 / \alpha}$.

Using the fact that these same assertions are valid for $1 / \alpha$ replacing $\alpha$ then establishes the theorem.

To verify (1), let $\varepsilon>0$ be given, with $\varepsilon<1 / 2$, and let $B=\{n \in \mathbb{N}:-\varepsilon<$ $\left.f_{1 / \alpha}(n)<\varepsilon\right\}$. We need to show $B \in h_{\alpha}(p)$. Pick $\delta>0$ such that $\delta<\varepsilon \cdot \alpha$ and $\delta<1 / 2$. Let $C=\left\{n \in \mathbb{N}:-\delta<f_{\alpha}(n)<\delta\right\}$. We show $h_{\alpha}[C] \subseteq B$. Let $n \in C$ and let $m=h_{\alpha}(n)$ so that $m-\delta<\alpha n<m+\delta$. Then $m / \alpha-\delta / \alpha<n<m / \alpha+\delta / \alpha$ so $n-\varepsilon<n-\delta / \alpha<m / \alpha<n+\delta / \alpha<n+\varepsilon$. This says $m \in B$ as required.

To verify (2), let $p, q \in Z_{\alpha}$. Let $A=\left\{n \in \mathbb{N}:-1 / 4<f_{\alpha}(n)<1 / 4\right\}$ and observe that $A \in p$ and $A \in q$. Suppose that $h_{\alpha}(p+q) \neq h_{\alpha}(p)+h_{\alpha}(q)$ and let $U$ and $V$ be disjoint open neighborhoods of $h_{\alpha}(p+q)$ and $h_{\alpha}(p)+h_{\alpha}(q)$ respectively. Pick 
$B \in p+q$ with $h_{\alpha}[\bar{B}] \subseteq U$. Pick $W$, a neighborhood of $h_{\alpha}(q)$, with $h_{\alpha}(p)+W \subseteq V$ and pick $C \in q$ with $h_{\alpha}[\bar{C}] \subseteq W$. Then $A \cap\{n \in \mathbb{N}: B-n \in p\} \cap C \in q$ so pick $n$ in this intersection. Now $h_{\alpha}(n) \in \mathbb{N}$, and $h_{\alpha}(p)+h_{\alpha}(n) \in V$ so pick a neighborhood $R$ of $h_{\alpha}(p)$ with $R+h_{\alpha}(n) \subseteq V$. Pick $D \in p$ with $h_{\alpha}[\bar{D}] \subseteq R$. Then $A \cap D \cap(B-n) \in p$ so pick $m \in A \cap D \cap B-n$. Then $h_{\alpha}(m)+h_{\alpha}(n) \in V$ and $h_{\alpha}(m+n) \in U$. We show that $h_{\alpha}(m)+h_{\alpha}(n)=h_{\alpha}(m+n)$, obtaining the desired contradiction. Let $k=h_{\alpha}(m)$ and $l=h_{\alpha}(n)$. Then $k-1 / 4<\alpha m<k+1 / 4$ and $l-1 / 4<\alpha n<l+1 / 4$ so $k+l-1 / 2<\alpha(m+n)<k+l+1 / 2$ so that $h_{\alpha}(m+n)=k+l=h_{\alpha}(m)+h_{\alpha}(n)$.

To verify (3), let $\varepsilon>0$ be given with $\varepsilon<1$ and $\varepsilon<\alpha / 2$. Let $B=$

$\left\{n \in \mathbb{N}:-\varepsilon<f_{\alpha}(n)<\varepsilon\right\}$. Then $B \in p$ so it suffices to show that $h_{1 / \alpha} \circ h_{\alpha}$ agrees with the identity on $B$. Let $n \in B$ be given and let $m=h_{\alpha}(n)$. Then $m-\varepsilon<n \alpha<m+\varepsilon$ so $m / \alpha-1 / 2<m / \alpha-\varepsilon / \alpha<n<m / \alpha+\varepsilon / \alpha<m / \alpha+1 / 2$ so $n-1 / 2<m / \alpha<n+1 / 2$. Thus $h_{1 / \alpha}(m)=n$ as required.

To verify (4), let $\varepsilon>0$ be given with $\varepsilon<1 / 2$, and let $B=\{n \in \mathbb{N}$ : $-\varepsilon<$ $\left.f_{1 / \alpha}(n)<0\right\}$. We need to show $B \in h_{\alpha}(p)$. Pick $\delta>0$ with $\delta<\varepsilon \cdot \alpha$ and $\delta<1 / 2$. Let $C=\left\{n \in \mathbb{N}: 0<f_{\alpha}(n)<\delta\right\}$. We show $h_{\alpha}[C] \subseteq B$. Let $n \in C$ and let $m=h_{\alpha}(n)$ so that $m<\alpha n<m+\delta$. Then $m / \alpha<n<m / \alpha+\delta / \alpha<m / \alpha+\varepsilon$ so $n-\varepsilon<m / \alpha<n$ and hence $m \in B$ as required.

The proof of (5) is essentially the same as that of (4).

For our next algebraic preliminary we deal with the notion of the smallest ideal of a compact left topological semigroup.

5.11. Definition. Let $S$ be a semigroup. Then $K(S)=\bigcup\{R: R$ is a minimal right ideal of $S\}$.

It is a fact that if $S$ is a compact left topological semigroup, then $K(S)$ is the smallest two sided ideal of $S$ and in fact $K(S)=\bigcup\{L: L$ is a minimal left ideal of $S\}$. (See for example [7], in particular Theorem 1.3.11.) Observe that $p$ is a minimal idempotent of $S$ if and only if $p$ is an idempotent and $p \in K(S)$. See the above reference also for unfamiliar algebraic facts cited in the proofs below.

5.12. Lemma. Let $(S,+)$ be a compact left topological semigroup and let $T$ be a compact subsemigroup of $S$ such that whenever $p \in T, q \in S$, and $p+q \in T$ one has $q \in T$.

(a) If $R$ is a minimal right ideal of $S$ and $R \cap T \neq \emptyset$, then $R \cap T$ is a minimal right ideal of $T$.

(b) If $K(S) \cap T \neq \emptyset$, then $K(T)=K(S) \cap T$.

Proof. (a) $R \cap T$ is a right ideal of $T$ so pick a minimal right ideal $R^{*}$ of $T$ with $R^{*} \subseteq R \cap T$. Pick $p \in R^{*}$. Then $R^{*}=p+T$ (since $p+T$ is a right ideal of $T$ ) and $R=p+S$. To see that $R \cap T \subseteq R^{*}$ let $r \in R \cap T$ and pick $q \in S$ with $r=p+q$. Then by assumption $q \in T$ so $r \in p+T=R^{*}$.

(b) Since $K(S) \cap T \neq \emptyset$ we have $K(S) \cap T$ is an ideal of $T$ so $K(T) \subseteq K(S) \cap T$. To see that $K(S) \cap T \subseteq K(T)$, let $p \in K(S) \cap T$ and pick a minimal right ideal $R$ of $S$ with $p \in R$. Then $R \cap T$ is a minimal right ideal of $T$ so $p \in K(T)$.

5.13. Theorem. Let $\alpha>0$. Then $K\left(Z_{\alpha}\right)=K(\beta \mathbb{N}) \cap Z_{\alpha}$.

Proof. All idempotents are in $Z_{\alpha}$ so $K(\beta \mathbb{N}) \cap Z_{\alpha} \neq \emptyset$. Given $p \in Z_{\alpha}$ and $q \in \beta \mathbb{N}$ with $p+q \in Z_{\alpha}$ we have $0=f_{\alpha}(p+q)=f_{\alpha}(p)+f_{\alpha}(q)=0+f_{\alpha}(q)$ so $q \in Z_{\alpha}$. Thus Lemma 5.12 applies. 


\section{Algebraic Results-iterated speCtra}

We begin by accumulating the main results from Sections 2 and 4. (These results all do have algebraic proofs as well.)

6.1. Theorem. Let $\alpha>0$, let $0 \leq \gamma<1$ with $\gamma>0$ if $\alpha$ is irrational, and let $A \subseteq \mathbb{N}$.

(a) If $A$ is a $\Delta^{*}$ set, then $g_{\alpha, \gamma}[A]=\{[n \alpha+\gamma]: n \in A\}$ is a $\Delta^{*}$ set.

(b) If $A$ is an $I P^{*}$ set, then $g_{\alpha, \gamma}[A]$ is an IP* set.

(c) If $A$ is a central ${ }^{*}$ set, then $g_{\alpha, \gamma}[A]$ is a central ${ }^{*}$ set.

(d) If $A$ is a central set, then $g_{\alpha, \gamma}[A]$ is a central set.

(e) If $A$ is an IP set, then $g_{\alpha, \gamma}[A]$ is an IP set.

(f) If $A$ is a $\Delta$ set, then $g_{\alpha, \gamma}[A]$ is a $\Delta$ set.

Proof. Theorems 2.3, 2.4, 4.1 and 4.3, Lemma 4.2, and Corollaries 2.6 and 2.8.

Theorem 6.1 together with Theorems 2.4 and 2.6 of [6] shows that spectra and even iterated spectra are combinatorially rich. Given $\alpha>0$ and $\gamma$ with $0 \leq \gamma<1$ (and $\gamma>0$ if $\alpha \notin Q$ ) one has for example that $\{[n \alpha+\gamma]: n \in \mathbb{N}\}$ is an $I P^{*}$ set.

Theorem 2.5 of [6] tells us that given any sequence $\left\langle x_{n}\right\rangle_{n=1}^{\infty}$ and any $I P^{*}$-set $A$, there is a sequence $\left\langle y_{n}\right\rangle_{n=1}^{\infty}$ with $\operatorname{FS}\left(\left\langle y_{n}\right\rangle_{n=1}^{\infty}\right) \subseteq \operatorname{FS}\left(\left\langle x_{n}\right\rangle_{n=1}^{\infty}\right)$ and $\operatorname{FS}\left(\left\langle y_{n}\right\rangle_{n=1}^{\infty}\right) \cup$ $\operatorname{FP}\left(\left\langle y_{n}\right\rangle_{n=1}^{\infty}\right) \subseteq A$. Since $I P^{*}$ sets are central* sets, $\{[n \alpha+\gamma]: n \in \mathbb{N}\}$ also contains solutions to any partition regular system of homogeneous linear equations. Now given $\alpha^{\prime}>0$ and $\gamma^{\prime}$ with $0 \leq \gamma^{\prime}<1$ (and $\gamma^{\prime}>0$ if $\alpha^{\prime} \notin \mathbb{Q}$ ) one has

$$
\left\{\left[[n \alpha+\gamma] \alpha^{\prime}+\gamma^{\prime}\right]: n \in \mathbb{N}\right\}=g_{\alpha^{\prime}, \gamma^{\prime}} \circ g_{\alpha, \gamma}[\mathbb{N}]
$$

and so is an $I P^{*}$ set and hence satisfies the same conclusions. The iteration can clearly continue any finite number of times.

It is a fact that for $\alpha>0$ and $0<\gamma<1$ the $\operatorname{sets}\{[n \alpha+\gamma]: n \in \mathbb{N}\}=g_{\alpha, \gamma}[\mathbb{N}]$ contain dynamical $I P^{*}$ sets (that is, sets of the form $\left\{n \in \mathbb{N}: \mu\left(A \cap T^{-n} A\right)>0\right\}$ where $\mu(A)>0$ ). On the other hand (see [6]) there exist $I P^{*}$ sets which do not contain dynamical $I P^{*}$ sets. Given any such set $A$, Theorem 6.1(a) tells us that $\{[n \alpha+\gamma]: n \in A\}$ is still an $I P^{*}$ set.

We restrict our attention to $0 \leq \gamma \leq 1$ for the following simple reasons. If $\alpha>2$ and say $1<\gamma<2$ we have $g_{\alpha, \gamma}[\overline{\mathbb{N}}] \cap \bar{g}_{\alpha, \gamma-1}[\mathbb{N}]=\emptyset$. Since the latter set is an $I P^{*}$ set, the former is not an $I P$ set. Likewise if $\alpha>2$ is rational, we have since $g_{\alpha, 0}[\mathbb{N}]$ is an $I P^{*}$ set that $g_{\alpha, 1}[\mathbb{N}]$ is not an $I P$ set. We shall see now that the sets $g_{\alpha, 0}[\mathbb{N}]$ and $g_{\alpha, 1}[\mathbb{N}]$ are both combinatorially rich if $\alpha$ is irrational. (And hence if $\alpha>2$, neither is an $I P^{*}$ set or even a central set.)

6.2. Theorem. Let $\alpha>0$ be irrational. There are minimal idempotents $p$ and $q$ of $(\beta \mathbb{N}, \cdot)$ such that every member of $p$ and every member of $q$ is additively central and such that $g_{\alpha, 0}[\mathbb{N}] \in p$ and $g_{\alpha, 1}[\mathbb{N}] \in q$.

Proof. Let $\delta=1 / \alpha$. We prove the assertion about $g_{\alpha, 0}[\mathbb{N}]$. The corresponding assertion about $g_{\alpha, a}[\mathbb{N}]$ uses $U_{\delta}$ and $X_{\delta}$ in place of $D_{\delta}$ and $Y_{\delta}$.

Let $M=\operatorname{cl}\{p: p$ is minimal idempotent of $(\beta \mathbb{N},+)\}$. We first show that $Y_{\delta} \subseteq$ $\overline{g_{\alpha, 0}[\mathbb{N}]}$. Indeed let $p \in Y_{\delta}$ and let $\varepsilon=\min \{1 / 2,1 / \alpha\}$. Let $B=\{n \in \mathbb{N}:-\varepsilon<$ $\left.f_{\delta}(n)<0\right\}$. Then $B \in p$. We show $B \subseteq g_{\alpha, 0}[\mathbb{N}]$. Given $n \in B$, let $m=h_{\delta}(n)$ so that $m-\varepsilon<\delta n<m$. Then $m \alpha-\varepsilon \alpha<n<m \alpha$ so $n<m \alpha<n+\varepsilon \alpha \leq n+1$ and hence $n=[m \alpha]$. It thus suffices to show that there is a minimal idempotent $p$ of $(\beta \mathbb{N}, \cdot)$ with $p \in M \cap Y_{\delta}$. 
By Theorem 5.6, $Y_{\delta}$ is a right ideal of $(\beta \mathbb{N}, \cdot)$. By [5, Theorem 5.4] $M$ is a right ideal of $(\beta \mathbb{N}, \cdot)$. It thus suffices to show that $Y_{\delta} \cap M \neq \emptyset$, for then $Y_{\delta} \cap M$ is a right ideal of $(\beta \mathbb{N}, \cdot)$ which thus contains an idempotent $p$ minimal in $(\beta \mathbb{N}, \cdot)$.

By Theorem $5.5, D_{\delta}$ is a left ideal of $(\beta \mathbb{N},+)$. Thus (see [7, Theorem 1.3.11]) $D_{\delta}$ contains an idempotent $r$ which is minimal in $(\beta \mathbb{N},+)$. Then immediately $r \in M$. Also $f_{\delta}(r)=0$ since $r+r=r$, so $r \in Y_{\delta}$, as required.

If $\alpha>2$ it is an easy exercise to show $g_{\alpha, 0}[\mathbb{N}] \cap g_{\alpha, 1}[\mathbb{N}]=\emptyset$. Consequently if $\alpha$ is also irrational, one has by Theorem 6.2 that there is an $I P^{*}$ set (namely $\mathbb{N}$ ) such that $g_{\alpha, 0}[\mathbb{N}]$ and $g_{\alpha, 1}[\mathbb{N}]$ are not central ${ }^{*}$.

In fact if $\alpha>1$ and irrational one can show as in the proof of Theorem 6.2 that $\overline{g_{\alpha, 0}[\mathbb{N}]} \cap X_{1 / \alpha}=\emptyset$ and $\overline{g_{\alpha, 1}[\mathbb{N}]} \cap Y_{1 / \alpha}=\emptyset$. (If $\varepsilon<\frac{\alpha-1}{\alpha}$ and $p \in X_{1 / \alpha}$, then $\left\{n \in \mathbb{N}: 0<f_{1 / \alpha}(n)<\varepsilon\right\} \in p$ and $\left\{n \in \mathbb{N}: 0<f_{1 / \alpha}(n)<\varepsilon\right\} \cap g_{\alpha, 0}[\mathbb{N}]=\emptyset$.) Since $U_{1 / \alpha}$ and $D_{1 / \alpha}$ are left ideals of $(\beta \mathbb{N},+)$, they contain minimal left ideals which then contain minimal idempotents which are then in $X_{1 / \alpha}$ and $Y_{1 / \alpha}$ respectively. (These facts are not as easy as the corresponding facts about right ideals, but see [7].) Consequently $g_{\alpha, 0}[\mathbb{N}]$ and $g_{\alpha, 1}[\mathbb{N}]$ are not central ${ }^{*}$ sets. Of course if $\alpha<1$ we have $g_{\alpha, 0}[\mathbb{N}]=g_{\alpha, 1}[\mathbb{N}]=\mathbb{N}$. Even then we shall see in Corollary 6.8 that there exists an $I P^{*}$ set $A$ with $g_{\alpha, 0}[A]$ and $g_{\alpha, 1}[A]$ not central*.

6.3. Theorem. Let $\alpha>0$ be irrational and let $A \subseteq \mathbb{N}$. If $A$ is a central ${ }^{*}$ set, then $g_{\alpha, 0}[A]$ and $g_{\alpha, 1}[A]$ are central sets.

Proof. We show that $g_{\alpha, 1}[A]$ is central. By Theorem $5.5 D_{\alpha}$ is a left ideal of $(\beta \mathbb{N},+)$ so by $\left[7\right.$, Theorem 1.3.11] $D_{\alpha}$ contains a minimal idempotent $p$. Since $p$ is an idempotent, $f_{\alpha}(p)=0$ so $p \in Y_{\alpha}$. Now $p \in K\left(Z_{\alpha}\right)$ so by Theorem 5.10 $h_{\alpha}(p) \in K\left(Z_{1 / \alpha}\right)$. By Theorem 5.1, $K\left(Z_{1 / \alpha}\right)=K(\beta \mathbb{N}) \cap Z_{1 / \alpha}$ and hence $h_{\alpha}(p)$ is a minimal idempotent. By Lemma $5.9 h_{\alpha}(p)=g_{\alpha, 1}(p)$. Since $A$ is central ${ }^{*}, A \in p$ so $g_{\alpha, 1}[A] \in g_{\alpha, 1}(p)$ and consequently $g_{\alpha, 1}[A]$ is central.

We will return to this topic in Corollary 6.8.

Because of the reduction in combinatorial strength from input to output in Theorem 6.3 we cannot simply iterate spectra with $\gamma=0$ or $\gamma=1$ at will.

For the remainder of this paper we consider spectra formed by a single iteration, namely sets of the form $g_{\alpha_{2}, \gamma_{2}} \circ g_{\alpha_{1}, \gamma_{1}}[A]=\left\{\left[\left[n \alpha_{1}+\gamma_{1}\right] \alpha_{2}+\gamma_{2}\right]: n \in A\right\}$. The case of a single iteration seems to be significantly simpler than that of multiple iterations. In fact we are able to solve this case in a rather complete fashion.

Compare the following result with Theorem 6.3.

6.4. Lemma. Let $\alpha>0$ be rational. If $\alpha>1$ then $g_{\alpha, 1}[\mathbb{N}]$ is not an IP set. If $\alpha \leq 1$ then there is some $a \in \mathbb{N}$ so that $g_{\alpha, 1}[\mathbb{N a} a$ is not an IP set.

Proof. Assume $\alpha=m / n$ where $m$ and $n$ are relatively prime integers. Assume first that $\alpha>1$, so $m>n$. We claim $g_{\alpha, 1}[\mathbb{N}] \cap \mathbb{N} m=\emptyset$ which suffices for the first assertion since $\mathbb{N} m$ is an $I P^{*}$ set. Indeed suppose we have $k, r \in \mathbb{N}$ with $g_{\alpha, 1}(r)=k m$. Then $k m \leq r \cdot(m / n)+1<k m+1$ so $k n \leq r+n / m<k n+n / m$. Since $r<k n$ we have $k n-1 \geq r \geq k n-n / m>k n-1$, a contradiction. For the second assertion let $a=2 n$. Then $g_{\alpha, 1}[\mathbb{N} a] \cap \mathbb{N} 2=\emptyset$.

We are precisely interested in $g_{\alpha_{2}, \gamma_{2}} \circ g_{\alpha_{1}, \gamma_{2}}[A]$ where $\alpha_{1}$ and $\alpha_{2}$ are irrational and $\gamma_{1}, \gamma_{2} \in\{0,1\}$. (Theorems 6.1 and 6.3 and Lemma 6.4 allow us to handle the other possibilities.) We obtain a necessary and sufficient condition for the image to be central which turns on the intersection of the sets $X_{\alpha}$ and $Y_{\delta}$. 
6.5. Lemma. Let $\alpha$ and $\delta$ be positive irrational numbers. The following statements are equivalent:

(a) $X_{\alpha} \cap X_{\delta} \neq \emptyset$.

(b) $Y_{\alpha} \cap X_{\delta} \neq \emptyset$.

(c) Either

(1) $1, \alpha, \delta$ are linearly independent over $\mathbb{Q}$ or

(2) there exist positive integers $m, r$, and $s$ with $m \alpha+r \delta=s$.

Proof. We show (a) $\Leftrightarrow$ (c), from which (b) $\Leftrightarrow$ (c) follows by interchanging $\alpha$ and $\delta$.

(a) $\Rightarrow(\mathrm{c})$. Assume (1) and (2) fail. Since (1) fails pick integers $m, r$, and $s$, not all 0 , with $m \alpha+r \delta=s$. Since $\alpha \notin \mathbb{Q}, r \neq 0$ and since $\delta \notin \mathbb{Q}, m \neq 0$. We may presume that $m>0$. Since (2) fails we must then have $r<0$ and so $m-r>0$. Let $\varepsilon=1 /(m-r)$. We have some $p \in Y_{\delta} \cap X_{\alpha}$ so $\left\{n \in \mathbb{Q}:-\varepsilon<f_{\delta}(n)<0\right.$ and $\left.0<f_{\alpha}(n)<\varepsilon\right\} \in p$. Pick $n$ with $-\varepsilon<f_{\delta}(n)<0$ and $0<f_{\alpha}(n)<\varepsilon$. Let $k=h_{\delta}(n)$ and $t=h_{\alpha}(n)$. Then $k-\varepsilon<n \delta<k$ and $t<\alpha n<t+\varepsilon$. Now $m>0$ and $r<0$ so $k r<n r \delta<k r-\varepsilon r$ and $m t<\alpha n m<m t+\varepsilon m$. Since $m \alpha+r \delta=s$ we thus have $m t<n(s-r \delta)<m t+\varepsilon m$ so that $m t+k r<n s<m t+k r+\varepsilon(m-r)=m t+k r+1$. Since $m, t, k, r, n$ and $s$ are integers this is a contradiction.

(c) $\Rightarrow$ (a) For each $\varepsilon>0$ let $A_{\varepsilon}=\left\{n \in \mathbb{N}: 0<f_{\alpha}(n)<\varepsilon\right.$ and $\left.-\varepsilon<f_{\delta}(n)<0\right\}$. It suffices to show each $A_{\varepsilon} \neq \emptyset$. For then pick $p \in \beta \mathbb{N}$ with $\left\{A_{\varepsilon}: \varepsilon>0\right\} \subseteq p$. One has $p \in Y_{\delta} \cap X_{\alpha}$.

Let $\varepsilon>0$ be given. If (1) holds apply Kronecker's Theorem [17, Theorem 442] to directly produce $n \in A_{\varepsilon}$. Therefore we assume (2) holds and pick positive integers $m, r$, and $s$ with $m \alpha+r \delta=s$. Since $\alpha \notin \mathbb{Q}$ pick by Kronecker's Theorem $n$ and $k$ in $\mathbb{N}$ with $k<n \alpha<k+\varepsilon /(m+r)$. Then $k r<n r \alpha<k r+\varepsilon r /(m+r)<k r+\varepsilon$ so $0<f_{\alpha}(n r)<\varepsilon$. Also $k m<n m \alpha=n s-n r \delta<k m+\varepsilon m /(m+r)<k m+\varepsilon$ so $n s-k m-\varepsilon<n r \delta<n s-k m$ and hence $-\varepsilon<f_{\delta}(n r)<0$. Therefore $n r \in A_{\varepsilon}$.

Note that condition (c) (2) of the following lemma includes the possibility that $s=0$, that is, that $\alpha / \delta$ is rational.

6.6. Lemma. Let $\alpha$ and $\delta$ be positive irrational numbers. The following statements are equivalent:

(a) $X_{\alpha} \cap X_{\delta} \neq \emptyset$.

(b) $Y_{\alpha} \cap Y_{\delta} \neq \emptyset$.

(c) Either

(1) $1, \alpha, \delta$ are linearly independent over $\mathbb{Q}$ or

(2) there exist integers $m, r$, and $s$ with $m>0, r<0$, and $m \alpha+r \delta=s$.

Proof. The proofs that (a) $\Leftrightarrow$ (c) and that (b) $\Leftrightarrow$ (c) are nearly identical to the proof that $(\mathrm{a}) \Leftrightarrow(\mathrm{c})$ in Lemma 6.5.

The following theorem determines precisely when a single iteration of spectra results in a combinatorially rich set. Recall that for any $a \in \mathbb{N}, \mathbb{N} a$ is an $I P^{*}$ set. Note that by Lemma 6.5 and 6.6 the conditions of Theorem 6.7 can be phrased as purely algebraic conditions on $\alpha_{1}$ and $\alpha_{2}$. Recall that a subset $A$ of $\mathbb{N}$ is an IP set if and only if there is some idempotent $p$ of $\beta \mathbb{N}$ with $A \in p$.

6.7. Theorem. Let $\alpha_{1}$ and $\alpha_{2}$ be positive irrationals and let $\gamma_{1}, \gamma_{2} \in\{0,1\}$. Consider statements (a), (b), and (c).

(a) $\gamma_{1}=\gamma_{2}$ and $Y_{1 / \alpha_{1}} \cap X_{\alpha_{2}} \neq \emptyset$,

(b) $\gamma_{1} \neq \gamma_{2}$ and $X_{1 / \alpha_{1}} \cap X_{\alpha_{2}} \neq \emptyset$, 
(c) $\gamma_{1} \neq \gamma_{2}$ and $\alpha_{2}<1$.

If any of (a), (b), or (c) holds and $A \subseteq \mathbb{N}$ is a central ${ }^{*}$ set, then $g_{\alpha_{2}, \gamma_{2}} \circ g_{\alpha_{1}, \gamma_{1}}[A]$ is central. If none of (a), (b) or (c) holds, there is some $a \in \mathbb{N}$ such that $g_{\alpha_{2}, \gamma_{2}} \circ$ $g_{\alpha_{1}, \gamma_{1}}[\mathbb{N a}]$ is not an IP set.

Proof. Assume (a) or (b) holds. There are four cases involved depending on the values of $\gamma_{1}$ and $\gamma_{2}$. We do the case $\gamma_{1}=\gamma_{2}=1$, the others being very similar. Since $Y_{1 / \alpha_{1}} \cap X_{\alpha_{2}} \neq \emptyset$ we have by Lemma 6.5 that $X_{1 / \alpha_{1}} \cap Y_{\alpha_{2}} \neq \emptyset$. Let $W_{2}=$ $X_{1 / \alpha_{1}} \cap Y_{\alpha_{2}}$ and let $V_{2}=Z_{1 / \alpha_{1}} \cap Z_{\alpha_{2}}$. Then $W_{2}=U_{1 / \alpha_{1}} \cap D_{\alpha_{2}} \cap V_{2}$. Since by assumption $W_{2} \neq \emptyset$ this says $W_{2}$ is a left ideal of $V_{2}$. Let $W_{1}=h_{1 / \alpha_{1}}\left[W_{2}\right]$ and $V_{1}=h_{1 / \alpha_{1}}\left[V_{2}\right]$. Then $W_{1}$ is a left ideal of $V_{1}$ and $V_{1}$ contains all idempotents of $\beta \mathbb{N}$. Consequently $V_{1} \cap K(\beta \mathbb{N}) \neq \emptyset$. Pick a minimal left ideal $L$ of $V_{1}$ with $L \subseteq W_{1}$. Then $L \subseteq K\left(V_{1}\right) \subseteq V_{1} \cap K(\beta \mathbb{N})$. Pick an idempotent $p \in L$. Now $h_{\alpha_{1}}(p) \in K\left(V_{2}\right) \subseteq$ $K\left(Z_{1 / \alpha_{1}}\right)=K(\beta \bar{N}) \cap Z_{1 / \alpha_{1}}$ by Theorem 5.13, so $h_{\alpha_{1}}(p) \in K(\beta \mathbb{N}) \cap Z_{\alpha_{2}}=K\left(Z_{\alpha_{2}}\right)$ so $h_{\alpha_{2}} \circ h_{\alpha_{1}}(p) \in K\left(Z_{1 / \alpha_{2}}\right) \subseteq K(\beta \mathbb{N})$ so $h_{\alpha_{2}} \circ h_{\alpha_{1}}(p)$ is a minimal idempotent. Now $p$ is a minimal idempotent and $A$ is a central ${ }^{*}$ set so $A \in p$ so $g_{\alpha_{2}, \gamma_{2}} \circ g_{\alpha_{1}, \gamma_{1}}[A] \in$ $g_{\alpha_{2}, \gamma_{2}} \circ g_{\alpha_{1}, \gamma_{1}}(p)$. It thus suffices to show that $g_{\alpha_{2}, \gamma_{2}} \circ g_{\alpha_{1}, \gamma_{1}}(p)=h_{\alpha_{2}} \circ h_{\alpha_{1}}(p)$. Now $p \in W_{1}=h_{1 / \alpha_{1}}\left[W_{2}\right] \subseteq h_{1 / \alpha_{1}}\left[X_{1 / \alpha_{1}}\right]=Y_{\alpha_{1}}$. Therefore $h_{\alpha_{1}}(p)=g_{\alpha_{1}, 1}(p)$. Also $h_{\alpha_{1}}(p) \in W_{2} \subseteq Y_{\alpha_{2}}$ so $h_{\alpha_{2}}\left(h_{\alpha_{1}}(p)\right)=g_{\alpha_{2}, 1}\left(h_{\alpha_{1}}(p)\right)=g_{\alpha_{2}, 1}\left(g_{\alpha_{1}, 1}(p)\right)$ as required.

Now assume that (c) holds. We may further assume that (b) does not hold so that $X_{1 / \alpha_{1}} \cap X_{\alpha_{2}}=\emptyset$. There are two nearly identical cases. We do the case $\gamma_{1}=0$ and $\gamma_{2}=1$. We know $Y_{\alpha_{2}}$ is a compact subsemigroup of $\beta \mathbb{N}$ which thus has idempotents. By Lemma 6.6 we have $Y_{\alpha_{2}} \cap Y_{1 / \alpha_{1}}=\emptyset$ so $Y_{\alpha_{2}} \cap X_{1 / \alpha_{1}} \neq \emptyset$. Thus we may proceed exactly as in the first case of this proof obtaining a minimal idempotent $p \in V_{1}=h_{1 / \alpha_{1}}\left[X_{1 / \alpha_{1}} \cap Y_{\alpha_{2}}\right]$ and concluding as there that $h_{\alpha_{2}} \circ h_{\alpha_{1}}(p)$ is a minimal idempotent. Now let $B=\left\{n \in \mathbb{N}:-1 / 2<f_{\alpha_{1}}(n)<0\right\}$. Since $p \in h_{1 / \alpha_{1}}\left[X_{1 / \alpha_{1}}\right]=Y_{\alpha_{1}}$ we have $B \in p$. Let $\varepsilon=1-\alpha_{2}$ (which is positive by assumption) and let $E=\left\{n \in \mathbb{N}:-\varepsilon<f_{\alpha_{2}}(n)<0\right\}$. Since $h_{\alpha_{1}}(p) \in Y_{\alpha_{2}}$ we have $E \in h_{\alpha_{1}}(p)$. Pick $D \in p$ with $h_{\alpha_{1}}[D] \subseteq E$. Since $p$ is a minimal idempotent we have $A \in p$. Thus $h_{\alpha_{2}} \circ h_{\alpha_{1}}[A \cap B \cap D] \in h_{\alpha_{2}} \circ h_{\alpha_{1}}(p)$ so it suffices to show that $h_{\alpha_{2}} \circ h_{\alpha_{1}}[A \cap B \cap D] \subseteq g_{\alpha_{2}, 1} \circ g_{\alpha_{1}, 0}[A]$. To this end let $w \in h_{\alpha_{2}} \circ h_{\alpha_{1}}[A \cap B \cap D]$. Pick $x \in A \cap B \cap D$ with $w=h_{\alpha_{2}}\left(h_{\alpha_{1}}(x)\right)$ and let $m=h_{\alpha_{1}}(x)$. Now $x \in B$ so $m-1 / 2<\alpha_{1} x<m$ so $g_{\alpha_{1}, 0}(x)=m-1$. We show that $w=g_{\alpha_{2}, 1}(m-1)$ so that $w \in g_{\alpha_{2}, 1} \circ g_{\alpha_{1}, 0}[A]$. Now $x \in D$ so $m \in E$ so (since $\left.w=h_{\alpha_{2}}(m)\right) w-\varepsilon<\alpha_{2} m<w$. Since $\varepsilon<1-\alpha_{2}$ we have $w-1<\alpha_{2}(m-1)<w$ so $w=g_{\alpha_{2}, 1}(m-1)$ as required.

Now assume none of (a), (b), or (c) holds. Assume first that $\gamma_{1}=\gamma_{2}$. The two cases are nearly identical. We do the case $\gamma_{1}=\gamma_{2}=0$. Pick $a \in \mathbb{N}$ with $a>2 / \alpha_{1}+2 /\left(\alpha_{1} \alpha_{2}\right)$. Let $C=g_{\alpha_{2}, 0} \circ g_{\alpha_{1}, 0}[\mathbb{N} a]$ and suppose we have an idempotent $p$ with $C \in p$. Let $q=h_{1 / \alpha_{2}}(p)$ and let $r=h_{1 / \alpha_{1}}(q)$. Then $q$ and $r$ are idempotents and therefore $\mathbb{N} a \in r$.

Consider first the possibility that $q \in Y_{\alpha_{2}}$. By Lemma $6.5 q \notin X_{1 / \alpha_{1}}$ so $q \in$ $Y_{1 / \alpha_{1}}$. Then $r \in X_{\alpha_{1}}$, so $q=h_{\alpha_{1}}(r)=g_{\alpha_{1}, 0}(r)$ so $g_{\alpha_{1}, 0}[\mathbb{N} a] \in q$. Also $p=$ $h_{\alpha_{2}}(q)=g_{\alpha_{2}, 1}(q)$ so $g_{\alpha_{2}, 1} \circ g_{\alpha_{1}, 0}[\mathbb{N} a] \in p$. By assumption $C \in p$ so pick $w \in$ $g_{\alpha_{2}, 1} \circ g_{\alpha_{1}, 0}[\mathbb{N} a] \cap g_{\alpha_{2}, 0} \circ g_{\alpha_{1}, 0}[\mathbb{N} a]$ and pick $x, y \in \mathbb{N} a$ with $w=g_{\alpha_{2}, 1}\left(g_{\alpha_{1}, 0}(y)\right)=$ $g_{\alpha_{2}, 0}\left(g_{\alpha_{1}, 0}(x)\right)$. Let $k=g_{\alpha_{1}, 0}(x)$ and $m=g_{\alpha_{1}, 0}(y)$. Then $k<\alpha_{1} x<k+1$ and $m<\alpha_{1} y<m+1$ so $\alpha_{2} k<\alpha_{1} \alpha_{2} x<\alpha_{2} k+\alpha_{2}$ and $\alpha_{2} m<\alpha_{1} \alpha_{2} y<\alpha_{2} m+\alpha_{2}$. Also $w=g_{\alpha_{2}, 1}(m)=g_{\alpha_{2}, 0}(k)$ so $w-1<\alpha_{2} m<w$ and $w<\alpha_{2} k<w+1$. Thus $\alpha_{1} \alpha_{2} y<\alpha_{2} m+\alpha_{2}<w+\alpha_{2}<\alpha_{2} k+\alpha_{2}<\alpha_{1} \alpha_{2} x+\alpha_{2}<\alpha_{2} k+2 \alpha_{2}<w+1+2 \alpha_{2}<$ $\alpha_{2} m+2+2 \alpha_{2}<\alpha_{1} \alpha_{2} y+2+2 \alpha_{2}$. Thus $-\alpha_{2}<\alpha_{1} \alpha_{2} x-\alpha_{1} \alpha_{2} y<2+\alpha_{2}$ so 
$-a<-1 / \alpha_{1}<x-y<2 /\left(\alpha_{1} \alpha_{2}\right)+1 / \alpha_{1}<a$. Since $x, y \in \mathbb{N} a$ this says $x=y$. Then $k=m$ while $w<\alpha_{2} k=\alpha_{2} m<w$, a contradiction.

Now we assume $q \notin Y_{\alpha_{2}}$ from which we conclude $q \in X_{\alpha_{2}}$. Since $X_{\alpha_{2}} \cap Y_{1 / \alpha_{1}}=$ $\emptyset$ we have $q \in X_{1 / \alpha_{1}}$. Then $r \in Y_{\alpha_{1}}$ so $q=g_{\alpha_{1}, 1}(r)$ so $g_{\alpha_{1}, 1}[\mathbb{N} a] \in q$. Let $\varepsilon=\min \left\{\alpha_{2}, 1 / 2\right\}$ and let $D=\left\{n \in \mathbb{N}: 0<f_{\alpha_{2}}(n)<\varepsilon\right\}$. Since $q \in X_{\alpha_{2}}$ we have $D \in q$. Also $p=g_{\alpha_{2}, 0}(q)$ so $g_{\alpha_{2}, 0}\left[g_{\alpha_{1}, 1}[\mathbb{N} a] \cap D\right] \in p$. Since by assumption $C \in p$, pick $w \in g_{\alpha_{2}, 0} \circ g_{\alpha_{1}, 0}[\mathbb{N} a] \cap g_{\alpha_{2}, 0}\left[g_{\alpha_{1}, 1}[\mathbb{N} a] \cap D\right]$. Pick $k \in g_{\alpha_{1}, 0}[\mathbb{N} a]$ and $m \in g_{\alpha_{1}, 1}[\mathbb{N} a] \cap D$ with $w=g_{\alpha_{2}, 0}(m)=g_{\alpha_{2}, 0}(k)$. Then $w<\alpha_{2} m<w+1$ and $w<\alpha_{2} k<w+1$. Pick $x, y \in \mathbb{N} a$ with $k=g_{\alpha_{1}, 0}(x)$ and $m=g_{\alpha_{1}, 1}(y)$. Then $k<\alpha_{1} x<k+1$ and $m-1<\alpha_{1} y<m$ so $\alpha_{2} k<\alpha_{1} \alpha_{2} x<\alpha_{2} k+\alpha_{2}$ and $\alpha_{2} m-\alpha_{2}<\alpha_{1} \alpha_{2} y<\alpha_{2} m$. Thus $\alpha_{1} \alpha_{2} y<\alpha_{2} m<w+1<\alpha_{2} k+1<\alpha_{1} \alpha_{2} x+1<$ $\alpha_{2} k+1+\alpha_{2}<w+2+\alpha_{2}<\alpha_{2} m+2+\alpha_{2}<\alpha_{1} \alpha_{2} y+2+2 \alpha_{2}$. Thus as above we conclude $x=y$. Since $k<\alpha_{1} x<k+1$ and $m-1<\alpha_{1} y<m$ we have $k=m-1$. Now $m \in D$ so $w<\alpha_{2} m<w+\varepsilon$. Now also $w<\alpha_{2} k=\alpha_{2}(m-1)<w+1$ so $w+\alpha_{2}<\alpha_{2} m<w+\varepsilon$ so $\alpha_{2}<\varepsilon$, a contradiction.

Finally assume that $\gamma_{1} \neq \gamma_{2}$ and neither (b) nor (c) holds. We shall do the case $\gamma_{1}=0$ and $\gamma_{2}=1$. In this case, since (b) does not hold we have $X_{1 / \alpha_{1}} \cap X_{\alpha_{2}}=\emptyset$ and by Lemma $6.6 Y_{1 / \alpha_{1}} \cap Y_{\alpha_{2}}=\emptyset$. Pick $a \in \mathbb{N}$ such that $a>2 / \alpha_{1}+2 /\left(\alpha_{1} \alpha_{2}\right)$. We let $C=g_{\alpha_{2}, 1} \circ g_{\alpha_{1}, 0}[\mathbb{N} a]$ and show $C$ supports no idempotents. Suppose instead we have an idempotent $p$ with $C \in p$. Let $q=h_{1 / \alpha_{2}}(p)$ and let $r=h_{1 / \alpha_{1}}(q)$. Then $q$ and $r$ are idempotents and therefore $\mathbb{N} a \in r$.

Consider first the possibility that $q \in X_{\alpha_{2}}$. Then $q \notin X_{1 / \alpha_{1}}$ while $q \in Z_{1 / \alpha_{1}}$, so $q \in Y_{1 / \alpha_{1}}$. Therefore $r \in X_{\alpha_{1}}$ and so $q=h_{\alpha_{1}}(r)=g_{\alpha_{1}, 0}(r)$ so $g_{\alpha_{1}, 0}[\mathbb{N} a] \in q$. Since $q \in X_{\alpha_{2}}$ we have $p=g_{\alpha_{2}, 0}(q)$ and therefore $g_{\alpha_{2}, 0} \circ g_{\alpha_{1}, 0}[\mathbb{N} a] \in p$. By assumption $C \in p$ so pick $w \in g_{\alpha_{2}, 1} \circ g_{\alpha_{1}, 0}[\mathbb{N} a] \cap g_{\alpha_{2}, 0} \circ g_{\alpha_{1}, 0}[\mathbb{N} a]$. Pick $x$ and $y$ in $\mathbb{N} a$ such that $w=g_{\alpha_{2}, 1}\left(g_{\alpha_{1}, 0}(y)\right)=g_{\alpha_{2}, 0}\left(g_{\alpha_{1}, 0}(x)\right)$. Let $k=g_{\alpha_{1}, 0}(x)$ and $m=g_{\alpha_{1}, 0}(y)$. Then $k<\alpha_{1} x<k+1$ and $m<\alpha_{1} y<m+1$. Also $w=g_{\alpha_{2}, 1}(m)=g_{\alpha_{2}, 0}(k)$ so $w-1<\alpha_{2} m<w$ and $w<\alpha_{2} k<w+1$. But these eight inequalities were shown to yield a contradiction during the treatment of the case $\gamma_{1}=\gamma_{2}=0$ and $q \in Y_{\alpha_{2}}$.

Now we assume $q \notin X_{\alpha_{2}}$. Since $q \in Z_{\alpha_{2}}$ we have $q \in Y_{\alpha_{2}}$. Therefore $q \notin Y_{1 / \alpha_{1}}$, and consequently $q \in X_{1 / \alpha_{1}}$. Therefore $r \in Y_{\alpha_{1}}$ so $q=h_{\alpha_{1}}(r)=g_{\alpha_{1}, 1}(r)$, and therefore $g_{\alpha_{1}, 1}[\mathbb{N} a] \in q$. Since $q \in Y_{\alpha_{2}}$ we have $p=g_{\alpha_{2}, 1}(q)$ so $g_{\alpha_{2}, 1}\left[g_{\alpha_{1}, 1}[\mathbb{N} a]\right] \in p$. By assumption $C \in p$ so pick $w \in g_{\alpha_{2}, 1} \circ g_{\alpha_{1}, 1}[\mathbb{N} a] \cap g_{\alpha_{2}, 1} \circ g_{\alpha_{1}, 0}[\mathbb{N} a]$. Pick $x$ and $y$ in $\mathbb{N} a$ with $w=g_{\alpha_{2}, 1}\left(g_{\alpha_{1}, 0}(x)\right)=g_{\alpha_{2}, 1}\left(g_{\alpha_{1}, 1}(y)\right)$ and let $k=g_{\alpha_{1}, 0}(x)$ and $m=g_{\alpha_{1}, 1}(y)$. As above we conclude that $x=y$ and hence $k=m-1$. But now $w-1<\alpha_{2} k<w$ and $w-1<\alpha_{2} m<w$ so $\alpha_{2}=\alpha_{2}(m-k)<1$, a contradiction.

We saw in Theorem 6.3 that if $\alpha$ is irrational and $A$ is a central* set then $g_{\alpha, 0}[A]$ and $g_{\alpha, 1}[A]$ are central sets. We show now that, even for $\alpha<1$, the conclusion cannot be strengthened to central ${ }^{*}$, even if the hypothesis is strengthened to $I P^{*}$, and the hypothesis cannot be weakened to central.

6.8. Corollary. Let $\alpha>0$ be irrational. There is an $I P^{*}$ set $A$ such that neither $g_{\alpha, 0}[A]$ nor $g_{\alpha, 1}[A]$ is central ${ }^{*}$. There are central sets $B$ and $C$ such that neither $g_{\alpha, 1}[B]$ nor $g_{\alpha, 0}[C]$ is an IP set.

Proof. Let $\alpha_{1}=\alpha$ and let $\alpha_{2}=1 / \alpha$. Then $Y_{1 / \alpha_{1}} \cap X_{\alpha_{2}}=\emptyset$. Note that in the proof of the second half of Theorem 6.7, only the fact that $a$ was sufficiently large was used. Pick $a$ sufficiently large so that neither $g_{\alpha_{2}, 1} \circ g_{\alpha_{1}, 1}[\mathbb{N} a]$ nor $g_{\alpha_{2}, 0} \circ g_{\alpha_{1}, 0}[\mathbb{N} a]$ is 
an $I P$ set and let $A=\mathbb{N} a$. If one had say that $g_{\alpha_{1}, 0}[A]$ were central ${ }^{*}$, Theorem 6.3 would guarantee that $g_{\alpha_{2}, 0} \circ g_{\alpha_{1}, 0}[A]$ is central.

Likewise one can get $a \in \mathbb{N}$ so that $g_{\alpha_{1}, 1} \circ g_{\alpha_{2}, 1}[\mathbb{N} a]$ and $g_{\alpha_{1}, 0} \circ g_{\alpha_{2}, 0}[\mathbb{N} a]$ do not support idempotents. Let $B=g_{\alpha_{2}, 1}[\mathbb{N} a]$ and $C=g_{\alpha_{2}, 0}[\mathbb{N} a]$. By Theorem $6.3 B$ and $C$ are central.

We conclude by showing in Theorem 6.10 that if both $\alpha_{2}$ and $\alpha_{1}$ are bigger than 1 we get an even stronger result than in the second half of Theorem 6.76.7.

6.9. Lemma. If $\alpha>1$ then $g_{\alpha, 1}[\mathbb{N}] \cap g_{\alpha, 0}[\mathbb{N}]$ is not an IP set.

Proof. Suppose we have an idempotent $p$ with $g_{\alpha, 1}[\mathbb{N}] \cap g_{\alpha, 0}[\mathbb{N}] \in p$. Let $\varepsilon=$ $1-1 / \alpha$ and let $B=\left\{n \in \mathbb{N}:-\varepsilon<f_{1 / \alpha}(n)<\varepsilon\right\}$. Then $B \in p$ so pick $n \in$ $g_{\alpha, 1}[\mathbb{N}] \cap g_{\alpha, 0}[\mathbb{N}] \cap B$. Pick $k, m \in \mathbb{N}$ such that $n=g_{\alpha, 1}(k)=g_{\alpha, 0}(m)$. Then $n-1 \leq \alpha k<n$ and $n \leq \alpha m<n+1$. Then $k<n / \alpha \leq k+1 / \alpha<k+1$ and $m-1<m-1 / \alpha<n / \alpha \leq m$ so $k=m-1$. Since $n \in B$ we have either $k<n / \alpha<k+\varepsilon$ or $k+1-\varepsilon<n / \alpha<k+1$. But $n / \alpha \leq k+1 / \alpha=k+1-\varepsilon$ so the latter case cannot hold. Then $m-1<n / \alpha<m-1+\varepsilon=m-1 / \alpha<n / \alpha$, again a contradiction.

6.10. Theorem. Let $\alpha_{1}$ and $\alpha_{2}$ be irrationals each bigger than 1 and let $\gamma_{1}, \gamma_{2} \in$ $\{0,1\}$. If either (a) $\gamma_{1}=\gamma_{2}$ and $Y_{1 / \alpha_{1}} \cap X_{\alpha_{2}}=\emptyset$ or (b) $\gamma_{1} \neq \gamma_{2}$ and $X_{1 / \alpha_{1}} \cap X_{\alpha_{2}}=\emptyset$, then $g_{\alpha_{2}, \gamma_{2}} \circ g_{\alpha_{1}, \gamma_{1}}[\mathbb{N}]$ is not an IP set.

Proof. Again there are four cases. This time we will do the case $\gamma_{1}=1$ and $\gamma_{2}=0$. We have $X_{1 / \alpha_{1}} \cap X_{\alpha_{2}}=\emptyset$. Suppose we have an idempotent $p$ with $g_{\alpha_{2}, 0} \circ g_{\alpha_{1}, 1}[\mathbb{N}] \in p$ and let $q=h_{1 / \alpha_{2}}(p)$. Assume first that $q \in Y_{\alpha_{2}}$. Then $p=h_{\alpha_{2}}(q)=g_{\alpha_{2}, 1}(q)$ so $g_{\alpha_{2}, 1}[\mathbb{N}] \in p$ while $g_{\alpha_{2}, 0} \circ g_{\alpha_{1}, 1}[\mathbb{N}] \subseteq g_{\alpha_{2}, 0}[\mathbb{N}]$ so $g_{\alpha_{2}, 0}[\mathbb{N}] \in p$. This contradicts Lemma 6.9.

Thus $q \notin Y_{\alpha_{2}}$. Then $q \in X_{\alpha_{2}}$ and since $X_{\alpha_{2}} \cap X_{1 / \alpha_{1}}=\emptyset, q \in Y_{1 / \alpha_{1}}$. Let $r=h_{1 / \alpha_{1}}(q)$. Then $r \in X_{\alpha_{1}}$ so $q=h_{\alpha_{1}}(r)=g_{\alpha_{1}, 0}(r)$ and hence $g_{\alpha_{1}, 0}[\mathbb{N}] \in q$. By Lemma 6.12 we have $g_{\alpha_{1}, 1}[\mathbb{N}] \notin q$ so $A=\mathbb{N} \backslash g_{\alpha_{1}, 1}[\mathbb{N}] \in q$. Since $q \in X_{\alpha_{2}}$, $p=h_{\alpha_{2}}(q)=g_{\alpha_{2}, 0}(q)$ so $g_{\alpha_{2}, 0}[A] \in p$. Also by assumption $g_{\alpha_{2}, 0} \circ g_{\alpha_{1}, 1}[\mathbb{N}] \in p$. But $\alpha_{2}>1$ so $g_{\alpha_{2}, 0}$ is one-to-one so $g_{\alpha_{2}, 0}[A] \cap g_{\alpha_{2}, 0} \circ g_{\alpha_{1}, 1}[\mathbb{N}]=\emptyset$, a contradiction.

\section{ACKNOWLEDGMENTS}

We wish to thank R. L. Graham, Yitzhak Katznelson, and Helge Tverberg for some helpful conversations and correspondence. We also thank the referee for suggestions which significantly improved the presentation.

\section{REFERENCES}

1. J. Auslander On the proximal relation in topological dynamics, Proc. Amer. Math. Soc. 11 (1960), 890-895. MR 29:1632

2. J. Baker and P. Milnes, The ideal structure of the Stone-Čech compactification of a group, Math. Proc. Cambridge Philos. Soc. 82 (1977), 401-409. MR 57:509

3. T. Bang, On the sequence $[n \alpha], n=1,2, \ldots$, Math. Scand. 5 (1957), 69-76. MR 19:1159h

4. V. Bergelson and N. Hindman, A combinatorially large cell of a partition of $\mathbb{N}$, J. Combin. Theory (Ser. A) 48 (1988), 39-52. MR 89m:04003

5. Nonmetrizable topological dynamics and Ramsey theory, Trans. Amer. Math. Soc. 320 (1990), 293-320. MR 90h:03046

6. - On IP* sets and central sets, Combinatorica 14 (1994), 269-277. CMP 95:05

7. J. Berglund, H. Junghenn, and P. Milnes, Analysis on semigroups, Wiley, New York, 1989. MR 91b: 43001 
8. M. Boshernitzan and A. Fraenkel, Nonhomogeneous spectra of numbers, Discrete Math. 34 (1981), 325-327. MR 82d:10077

9. R. Ellis, A semigroup associated with a transformation group, Trans. Amer. Math. Soc. 94 (1960), 272-281. MR 23:A961

10. Lectures on topological dynamics, New York, Benjamin, 1969. MR 42:2463

11. A. Fraenkel, Complementary systems of integers, Amer. Math. Monthly 84 (1977), 114-115. MR 55:2825

12. H. Furstenberg, Recurrence in ergodic theory and combinatorial number theory, Princeton Univ. Press, Princeton, NJ, 1981. MR 82j:28010

13. H. Furstenberg and B. Weiss, Simultaneous diophantine approximation and IP-sets, Acta Arith. 49 (1988), 413-426. MR 89f: 11097

14. 61-85. MR 80g:05009

15. R. Graham, On a theorem of Uspensky, Amer. Math. Monthly 70 (1963), 407-409. MR 26:6062

16. R. Graham, S. Lin, and C. Lin, Spectra of numbers, Math. Magazine 51 (1978), 174-176. MR 58: 10808

17. G. Hardy and E. Wright, An introduction to the theory of numbers, Oxford Univ. Press, Oxford, 1979. MR 81i:10002

18. N. Hindman, Finite sums from sequences within cells of a partition of $\mathbb{N}$, J. Combin. Theory (Ser. A) 17 (1974), 1-11. MR 50:2067

19. 19-32. MR 80b:10022

20. Summable ultrafilters and finite sums, Logic and Combinatorics, (S. Simpson, ed.), Contemporary Math., vol. 65, Amer. Math. Soc. Providence, RI, 1987, pp. 263-274. MR 88h:03070

21. The existence of certain ultrafilters on $\mathbb{N}$ and a conjecture of Graham and Rothschild, Proc. Amer. Math. Soc. 36 (1972), 341-346. MR 46:7041

22. _ Ultrafilters and combinatorial number theory, Number Theory Carbondale 1979, (M. Nathanson, ed.), Lecture Notes in Math., vol. 751, Springer, 1979, pp. 119-184. MR 81m:10019

23. N. Hindman and J. Pym, Free groups and semigroups in $\beta \mathbb{N}$, Semigroup Forum 30 (1984), 177-193. MR 86c:22002

24. J. Kelley, General topology, Van Nostrand, New York, 1955. MR 16:1136c

25. B. Kra, A dynamical approach to central sets and iterated spectra of numbers, Abstracts Amer. Math. Soc. 13 (1992), 294.

26. J. Lawson and A. Lisan, Transitive flows: a semigroup approach, Mathematika 38 (1991), 348-361. MR 93c:22003

27. I. Niven, Diophantine approximations, Interscience, New York, 1963. MR 26:6120

28. I. Schoenberg, Mathematical time exposures, Mathematical Association of America, Washington, DC, 1982. MR 85b:00001

29. T. Skolem, On certain distributions of integers in pairs with given differences, Math. Scand. 5 (1957), 57-68. MR 19:1159g

30. — Über einige Eigenschaften der Zahlenmengen $[\alpha n+\beta]$ bei irrationalem $\alpha$ mit einleitenden Bemerkungen über dinige kombinatorische probleme, Norske Vid. Selsk. Forh. 30 (1957), 42-49. MR 19:1159i

31. J. Strutt (Lord Rayleigh), The theory of sound, Macmillan, London, 1977; Reprinted, Dover, New York, 1945.

32. J. Uspensky, On a problem arising out of a certain game, Amer. Math. Monthly 34 (1927), 516-521.

33. B. van der Waerden, Beweis einer Baudetschen Vermutung, Nieuw Arch. Wisk. 19 (1927), 212-216.

Department of Mathematics, Ohio State University, Columbus, Ohio 43210-1328

E-mail address: vitaly@math.ohio-state.edu

Department of Mathematics, Howard University, Washington, D.C. 20059-0001

E-mail address: nhindman@aol.com

Department of Mathematics, Hebrew University of Jerusalem, Jerusalem, Israel

E-mail address: bryna@math.nuy.ac.il 